\title{
Flexible Brain Transitions Between Hierarchical Network Segregation and Integration Predict Human Behavior
}

\section{Rong Wang}

XI'an University of Science and Technology https://orcid.org/0000-0001-8599-9850

\section{Xiaoli Su}

Xi'an University of Science and Technology

\section{Zhao Chang}

Xi'an University of Science and Technology

\section{Ying Wu}

Pan Lin ( $\nabla$ tiger.lin9906@gmail.com )

hunan normal university

Article

Keywords: hierarchically, phenotype

Posted Date: November 2nd, 2020

DOI: https://doi.org/10.21203/rs.3.rs-94060/v1

License: (c) (1) This work is licensed under a Creative Commons Attribution 4.0 International License. Read Full License 


\title{
Flexible brain transitions between hierarchical network segregation and integration predict human behavior
}

\author{
Rong Wang ${ }^{1,2,3}$, Xiaoli Su${ }^{1}$, Zhao Chang ${ }^{1}$, Ying Wur ${ }^{2,3,4^{*}}$, Pan $\operatorname{Lin}^{5 *}$
}

${ }^{1}$ College of Science, Xi'an University of Science and Technology, Xi'an, 710054 China.

${ }^{2}$ State Key Laboratory for Strength and Vibration of Mechanical Structures, Xi'an Jiaotong University, Xi'an, 710049 China.

${ }^{3}$ School of Aerospace Engineering, Xi' an Jiaotong University, Xi'an, 710049 China.

${ }^{4}$ National Demonstration Center for Experimental Mechanics Education, Xi'an Jiaotong University, Xi'an, 710049 China.

${ }^{5}$ Department of Psychology and Cognition and Human Behavior Key Laboratory of Hunan Province, Hunan Normal University, Hunan, China.

*Corresponding authors: wying36@mail.xjtu.edu.cn (YW); tiger.lin9906@gmail.com (PL)

\begin{abstract}
Cognition involves locally segregated and globally integrated processing. This process is hierarchically organized, linking to evidence from hierarchical modules in brain networks. However, it remains a mystery how flexible transitions between these hierarchical processes are associated with human behavior. Here, we used a multisource interference task and measured hierarchical segregation and integration across multiple levels. Our results show more flexible transitions between segregated and integrated brain states in the task state. Crucially, brain flexibility in resting and task states was associated with human behavior. To achieve a better performance, the resting brain is optimized to be more flexible, such that it is prepared to more efficiently switch into a task state where the brain needs less flexibility to stably perform the task. Our hierarchical modular analysis was more effective in detecting the alterations in functional organization and the phenotype of human behavior than graph-based network measures at a single level.
\end{abstract}




\section{Introduction}

The brain is a complex network consisting of separate functional subsystems ${ }^{1}$. Successful cognitive behaviors require not only the relatively isolated communication within subsystems, i.e., functional segregation, but also the effectively global interaction between them, i.e., functional integration $^{2,3}$. Recent studies have suggested that the functional segregation and integration of complex brain networks are associated with brain cognitive functions ${ }^{4-7}$. For example, higher segregation within the brain network has been linked to motor execution, and higher integration has been shown to underlie working memory ${ }^{8}$. In fact, complex brain networks dynamically reconfigure to adapt to the environment to flexibly support variable cognitive demands ${ }^{8,9}$. Dynamic brain network analyses can detect the temporal evolution in brain functional patterns and are believed to effectively capture relevant features of cognitive behaviors ${ }^{10-12}$. In principle, flexible transitions in brain network organization between segregated and integrated states support the efficient communication of brain network subsystems across local and global scales, which is essential for successful cognitive performance. Despite great progress in understanding the dynamic transition between segregation and integration at rest and during tasks $8,9,13,14$, how cognitive behaviors are associated with dynamic reconfigurations in large-scale brain functional organization is still not clear.

Complex brain networks display adaptive modular structures across time ${ }^{13,15}$ that involve strong functional connectivity (FC) within modules and relatively weak connectivity between them. This modular feature allows for an integrated process within modules and segregation between them ${ }^{16,17}$. Indeed, module-based network measures offer a powerful approach for characterizing segregation and integration in brain functional organization. Many groups have explored the relationship between modules of brain networks, cognitive functions and mental disorders $8,9,13,16,18-20$. However, brain networks have a deeper signature of hierarchical modules within these structural and functional organizations ${ }^{21-25}$, where small modules are successively embedded within larger modules. This hierarchical organization provides several promising advantages regarding brain dynamics, including greater robustness, adaptivity and functional diversity $21,23,26$. The classic modular partition based on the modularity maximization method or previously defined modular partitions (e.g., functional subsystems) is performed at a single level 
and is incapable of revealing the hierarchical modular organization ${ }^{2,27,28}$, which limits further inference about how the hierarchical segregated and integrated processes shape an individual's cognitive abilities. Recently, a nested-spectral partition (NSP) method based on eigenmodes was found to effectively detect hierarchical modules in brain networks ${ }^{21}$, and a concept of hierarchically nested segregation and integration was proposed based on these hierarchical modules. However, whether a hierarchical modular analysis can effectively detect more specific alterations in functional organization during task performance and how the brain enables the flexible transition between hierarchically segregated and integrated states to dynamically adapt to cognitive demands are still unclear.

In this study, we explored whether brain characteristics of flexible transitions between hierarchical segregation and integration during rest and task are associated with human cognitive behavior. We addressed this question using resting- and task-state functional magnetic resonance imaging (fMRI) data and a behavioral measure of response time (RT) by using a multisource interference task (MSIT), which reliably activates the localized cingulo-frontal-parietal cognitive network and is a useful task to study normal human cognition and psychiatric pathophysiology ${ }^{29}$. Using the NSP method to detect hierarchical modules in brain $\mathrm{FC}$ networks ${ }^{21}$, we first defined hierarchical segregation and integration and investigated specific alterations at the local and global scales in the task state. We then compared the temporally flexible transition between hierarchically segregated and integrated states in resting and task states. Finally, we tested whether the brain measures based on hierarchical modules captured more intrinsic phenotypes of human cognitive behavior than graph-based network measures at a single level.

We found that the brain resolved into a greater number of hierarchical modules resulting in higher segregation in the task state on the global scale and in most brain subsystems; conversely, the task state required a stronger integrated process in the brain control subsystem. The flexible transition between segregated and integrated states was significantly enhanced during task performance at the global and local scales. Furthermore, flexible brain transitions were an apparent biomarker for human cognitive behavior. Functional flexibility in the whole-brain network and default mode network (DMN) subsystem during rest was positively correlated with cognitive performance, and there were significantly negative correlations with the whole-brain network, dorsal attention, 
limbic and visual subsystems in the task state. Meanwhile, alterations in functional flexibility between the task and resting states in the whole-brain network and most functional subsystems were robustly negatively related to cognitive performance. Crucially, these relationships to cognitive performance were not significant for graph-based network measures at a single level, suggesting the effectiveness of our method to study segregation and integration based on hierarchical modules in brain FC networks. Our results showed that flexible transitions between hierarchical segregation and integration during rest maintained an intrinsic ability to flexibly support successful cognitive task performance. In contrast, better human performance required stability in functional organization during task performance, which was achieved by effectively reconfiguring the functional organization in the transition from the resting to task states.

\section{Results}

Eighteen healthy participants were recruited to perform the MSIT according to the standard protocol $^{29-31}$. RTs were recorded by the computer, and the coefficient of variation in task performance, that is, $\mathrm{RT} /(\operatorname{Stdev}(\mathrm{RT}))$, was chosen as the primary behavioral measure of interest ${ }^{32}$. The brain was parcellated into 90 regions to construct dynamic FC networks with a sliding time window method, and negative connectivity was excluded. The functional modes (i.e., eigenvalues $\Lambda$ and eigenvectors $U$ ) were obtained from the eigenvalue decomposition in $\mathrm{FC}$ matrices, which were sorted in the descending order from $\Lambda$. Given the noise and finite size of fMRI data, few eigenvalues had negative values and were set as zero.

\section{Hierarchical modular organization in brain functional networks}

The NSP method successively detected the hierarchical modules in brain FC networks from low-order functional modes to high-order ones. Briefly, the group-averaged FC networks had the largest module (i.e., whole-brain network), and this was referred to as the first level, which corresponds to the first eigenvector with the same sign for each region (Figures 1a, b). In the second level, FC networks were partitioned into two large modules that corresponded to positive and negative signs in the second eigenvector. Each module in the second level was subdivided into two modules in the third level based on the negativity and positivity of eigenvector values (Figures 1a, b). The FC networks can be modularly partitioned into multiple levels with increasing order of functional modes, wherein the connection density and average link weights within large 
modules were clearly higher than those between modules at the same level (Figure 1a), indicating that the functional modes reflected the hierarchical modular partition in brain FC networks, especially low-order modes corresponding to pronounced modular levels.
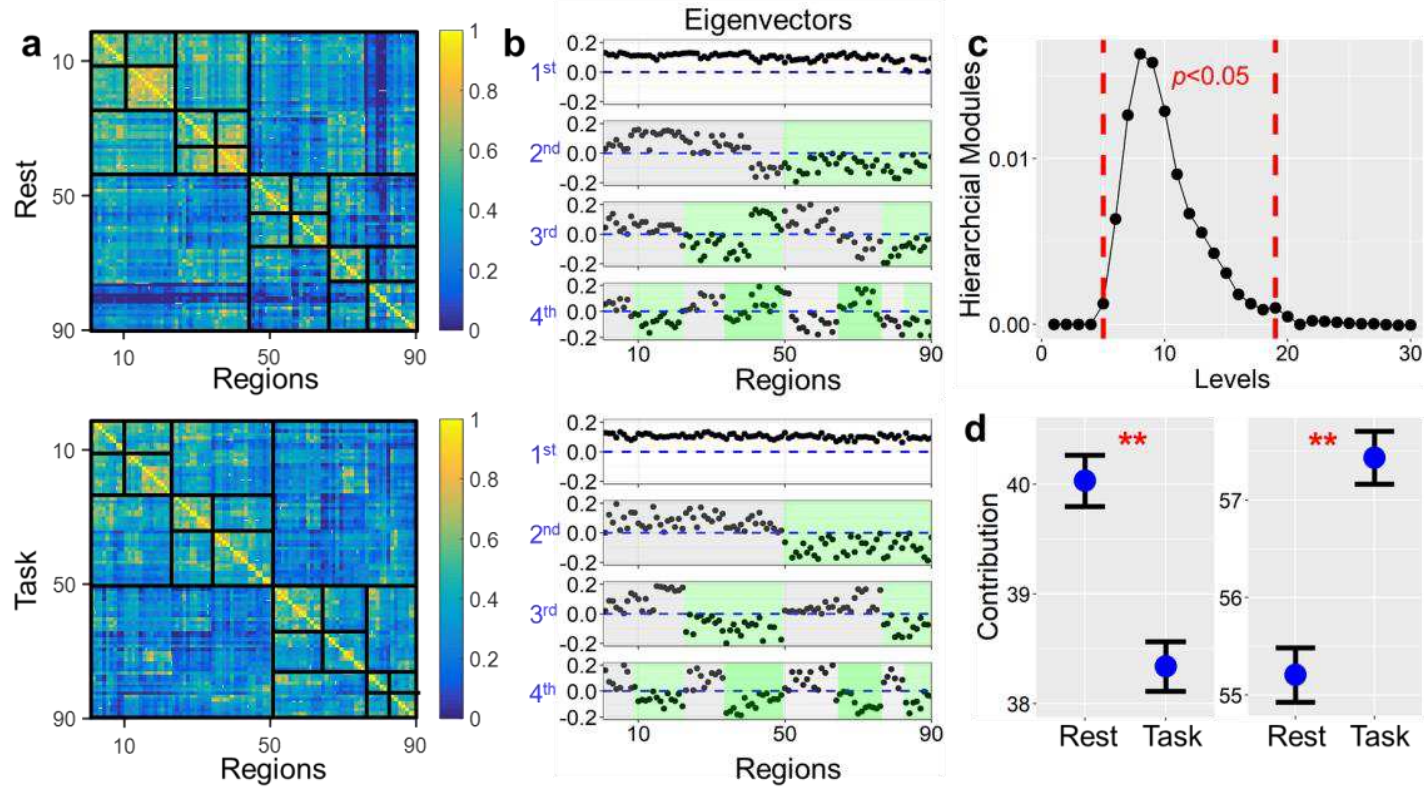

Figure 1. Hierarchical modular properties of brain functional networks. (a) Hierarchical modular structures of group-averaged FC matrices in resting (upper panel) and task (lower panel) states, detected by the NSP method based on the negativity and positivity of eigenvector values in each level ${ }^{21}$. The black lines represent the boundaries of hierarchical modules, and the regions were reordered during the NSP process (see Methods). (b) The hierarchical modular partition in the first four functional modes of FC networks in resting and task states, wherein the modules in each level are marked by gray and green colors. (c) The difference in module number between task and resting states in each level across all subjects, where the module number is significantly increased from the $5^{\text {th }}$ to $19^{\text {th }}$ levels (red range; two-sample t-test, $p<0.05$ ). (d) The comparison of the contribution (i.e., eigenvalue) in the first level (left panel) and that summed from the $2^{\text {nd }}$ to $90^{\text {th }}$ levels (right panel) in resting and task states. Here, “**” represents $p<0.01$ (two-sample t-test).

\section{Alterations in hierarchical segregation and integration in the task state}

Hierarchical modular detection by the NSP method includes the physical foundation concept that regions within a module are more likely to be coactivated to produce functional integration, and regions between modules are oppositely activated to generate segregation ${ }^{21}$. The first level contains one module corresponding to the whole-brain network, wherein all regions with the same 
sign of eigenvector values are cooperatively activated to produce the global integration. This globally coactivated pattern is crucial for communication between regions across the whole-brain network and requires the highest contribution (i.e., the largest eigenvalue, see Supplementary Figure 1), which is intrinsically constrained by the homogeneous structural pattern and is often used to reflect global synchronization ${ }^{21,33,34}$. As the level increased, large modules were divided into small modules, and the module number increases (Figure 1b and Supplementary Figure 2), which produced a stronger segregation of processes. These patterns supported more localized communication among regions within small modules at the high-order levels and thus required less contribution (i.e., smaller eigenvalues; see Supplementary Figure 1).

We observed that the contribution in the first level with one module was significantly decreased in the task state (Figure 1d, left panel), indicating a decreased degree of global integration in brain FC networks. Meanwhile, the module number was significantly increased at specific levels (from the $5^{\text {th }}$ to $19^{\text {th }}$ levels, see Figure $1 \mathrm{c}$ and Supplementary Figure 2), indicating that the brain recruited more hierarchical modules in FC networks during task performance. In addition, in the high-order levels (i.e., from $2^{\text {nd }}$ to $N^{\text {th }}$ ), the total contribution was significantly enhanced (Figure $1 \mathrm{~d}$ and Supplementary Figure 1), revealing that the levels with increased modules were more dominant in the task state. These results showed that the brain generates less global integration and more hierarchical segregation to match the functional demand of cognitive tasks.

To further confirm the above results, we proposed a measure of hierarchical segregation and integration by multiplying the contribution to the corresponding module number (i.e., weighted modules $H_{i}$; see Eq. 1). This measure reflects the domination of each level to global segregation and integration (see Methods). Consequently, the sum of $H_{i}$ from all levels considered the global-scaled integrated process and local-scaled segregated process across multiple levels and thus measured the hierarchical segregation and integration in FC networks (i.e., $H_{F}$; see Eq. 2). While the higher-order modes (i.e., from $2^{\text {nd }}$ to $N^{\text {th }}$ ) were more dominated with larger module numbers and contributions, the FC network was sparely connected and preferred segregation (Figure 2a), with higher $H_{i}$ in these levels and higher $H_{F}$ (Figure 2b). Otherwise, the first level corresponding to global integration was dominant, the FC network was densely connected and 
$H_{F}$ was small (Figures 2a, b). Thus, $H_{F}$ was negatively correlated to integration, with smaller values predicting higher integration (Figure 2b).

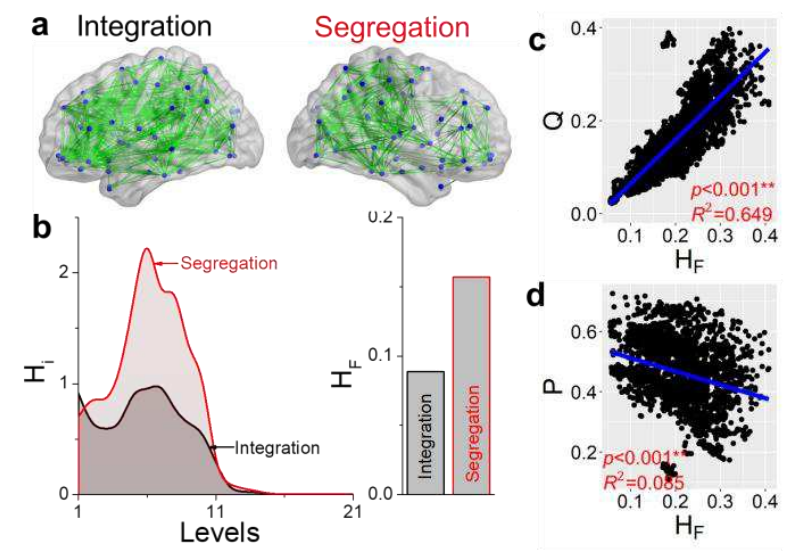

Figure 2. Hierarchical segregation and integration in brain functional organizations. (a)

Visualizations of two binarized FC networks in integrated and segregated states using the BrainNet Viewer ${ }^{35}$. (b) Schematic diagram for the definition of hierarchical segregation and integration. In the FC network, each level generates the segregation and integration, which is measured by the weighted modules (i.e., $H_{i}$ ). While the segregated levels with large modules are significantly dominant, the FC network shows higher segregation and sparse connectivity, as shown in a, accompanied by larger $H_{F}$. (c) The correlations of $H_{F}$ with modularity $(Q)$ and (d) with participation coefficient $(P)$, which were calculated from FC networks across all temporal windows and subjects in the resting state (see Supplementary Figure 3 for a similar result during the task state).

To test the reasonableness of the hierarchical segregation and integration (i.e., $H_{F}$ ), we computed the graph-based network measures of modularity and participation coefficient (see Eqs. 4, 5), which have been widely used to reflect functional segregation and integration in brain FC networks $3,19,20,36,37$. As expected, $H_{F}$ was positively correlated with the modularity and was negatively related to the participation coefficient (Figures $2 \mathrm{c}, \mathrm{d}$ ). This phenomenon was observed in the resting and task states (Figures $2 \mathrm{c}, \mathrm{d}$ and Supplementary Figure 3), indicating the effectiveness of $H_{F}$ in measuring functional segregation and integration in brain FC networks. However, it should also be noted that the graph-based network measures were highly variable for a similar $H_{F}$ (Figures 2c, d and Supplementary Figure 3), indicating that $H_{F}$ based on hierarchical levels may detect more individual properties in deeper levels than the modularity and 
participation coefficient at a single level, especially for brains in a segregated state (i.e., higher $\left.H_{F}\right)$.

a

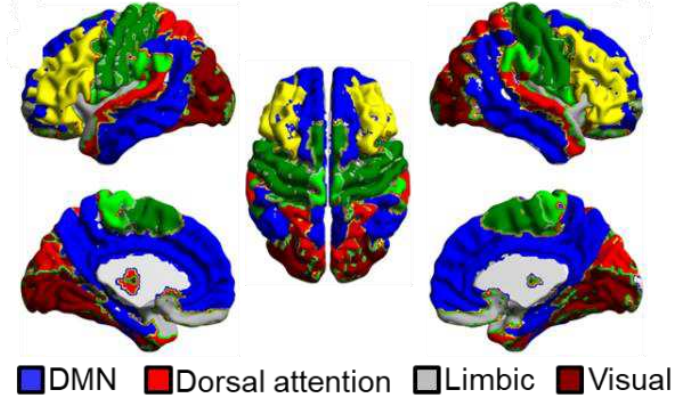

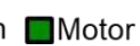

b
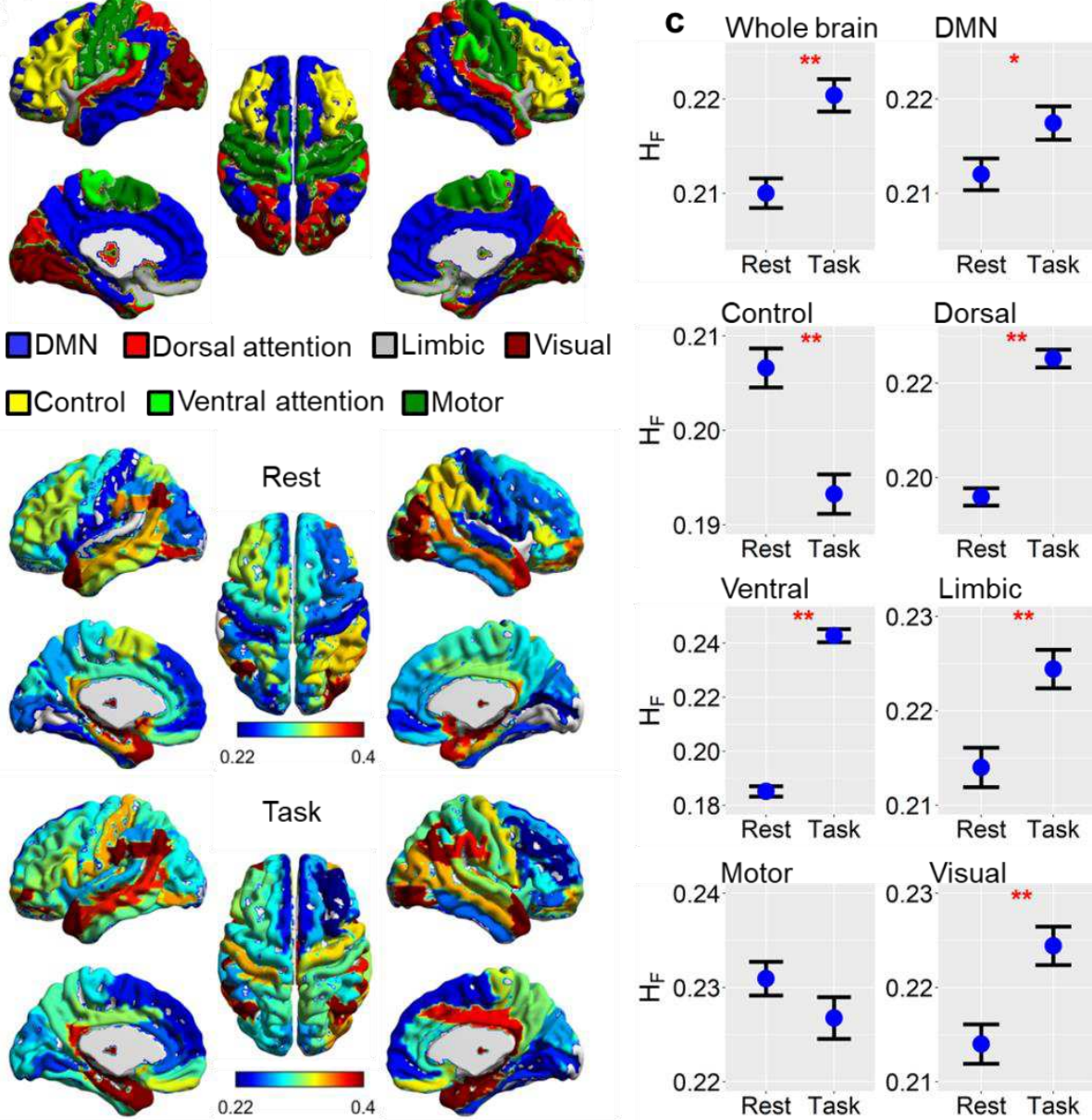

포 0.20

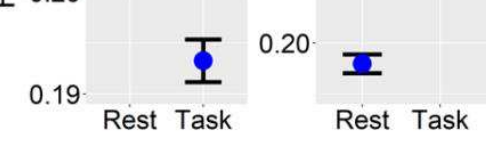

Ventral

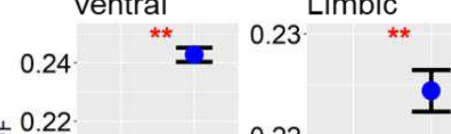

노 0.22

0.20

0.18

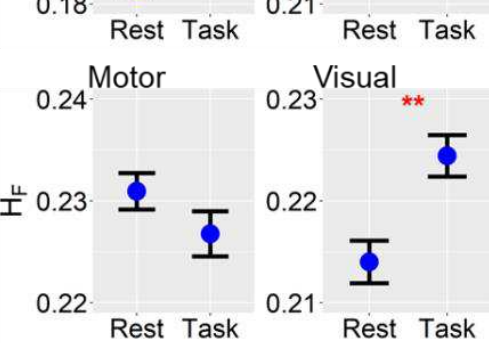

Figure 3. Alterations in hierarchical segregation and integration in the task state. (a) Brain surface mappings of seven functional subsystems using BrainNet Viewer ${ }^{35}$. (b) The hierarchical segregation and integration in each region (i.e., $H_{F}^{j}$, see Eq. 3) in resting (upper panel) and task (lower panel) states, which is averaged across all time windows and subjects. (c) The comparison of $H_{F}$ in the whole-brain network and seven subsystems in resting and task states, wherein "**, is a significant difference (two-sample t-test, $p<0.05$, FDR corrected) and “**” represents $p<0.01$.

By affecting the hierarchical modular properties in FC networks, MSIT requires more segregated processes at multiple levels (Figures 1c, d), as further confirmed by the significantly increased $H_{F}$ at the global scale (see Figure 3c). This result was consistent with observations obtained with the modularity and participation coefficient (Supplementary Figure 4), indicating that the brain needed more segregated communication among regions to process neural information during task 
performance. However, the hierarchical segregation and integration in each region (i.e., $H_{F}^{j}$; see Eq. 3) showed significant heterogeneity (Figure 3b). While most cortical regions had increased $H_{F}^{j}$ and contributed to higher segregation, prefrontal and posterior parietal regions have decreased $H_{F}^{j}$ and were recruited to generate higher integration (Figure 3b). Thus, we divided the brain into seven functional subsystems (Figure 3a), including the DMN, control, dorsal attention, ventral attention, limbic, motor and visual subsystems ${ }^{1,38}$, and we tested whether different subsystems had specific responses to MSIT. The $H_{F}^{j}$ values of the regions involved in a subsystem were averaged and referred to as the hierarchical segregation and integration of the subsystem. It was observed that all functional subsystems were sensitive to the task with the exception of the motor subsystem (Figure 3c). Meanwhile, the significantly altered subsystems also had specific responses to the task. The DMN, dorsal attention, ventral attention, limbic and visual subsystems had significantly increased $H_{F}$ in the task state (Figure 3c), indicating a higher segregated process in these subsystems. In contrast, the control subsystem showed decreased $H_{F}$ during the task state, reflecting a stronger integrated process. Thus, while the brain needed a more segregated process across the whole network to perform the task, the control subsystem maintained higher integration to effectively handle cognitive demand during the task.

\section{Greater flexibility in transitions between segregated and integrated states during the task}

The functional organization of the brain is dynamically reconfiguring during rest and task performance, wherein the flexible transition between segregated and integrated states was shown to be a significant signature for many cognitive abilities ${ }^{13,39-41}$. When all functional subsystems are temporally cooperatively activated, brain functional organization is in an integrated state with low $H_{F}$ and modularity (see Figures $4 \mathrm{a}, \mathrm{b}$ ). In contrast, when functional subsystems are weakly activated and operating in a relatively isolated fashion, the brain is in a segregated state with high $H_{F}$ and modularity (Figures $4 \mathrm{a}, \mathrm{b}$ ). This dynamically flexible transition between segregated and integrated states is classically measured by modularity ${ }^{13}$, but it also can be effectively reflected by changes in $H_{F}$. To measure this flexible transition in dynamic functional organization, we defined the standard variance in the $H_{F}$ time series as functional flexibility $F$ (see Figure $4 \mathrm{~b}$ ), and a higher $F$ reflects a stronger transition between segregated and integrated states. 
Apparently, the functional organization during the task more flexibly transitioned between segregated and integrated states than during rest (Figure 4a), as confirmed by the significantly increased functional flexibility (see Figure $4 \mathrm{c}$ for the whole-brain network), indicating that brain functional subsystems are more isolated and operated to flexibly transition between segregated and integrated states during the task state. Meanwhile, increased functional flexibility in the task state was also observed in all functional subsystems except for the limbic subsystem (Figure 4c), indicating more flexible transitioning between segregated and integrated states at the local and global scales. In addition, the above results were not observed, when functional flexibility was measured by the standard variance in modularity or participation coefficient (Supplementary Figure 5), further suggesting that $H_{F}$ values based on hierarchical modules were more sensitive to properties of dynamic functional organization than graph-based network measures at a single level.
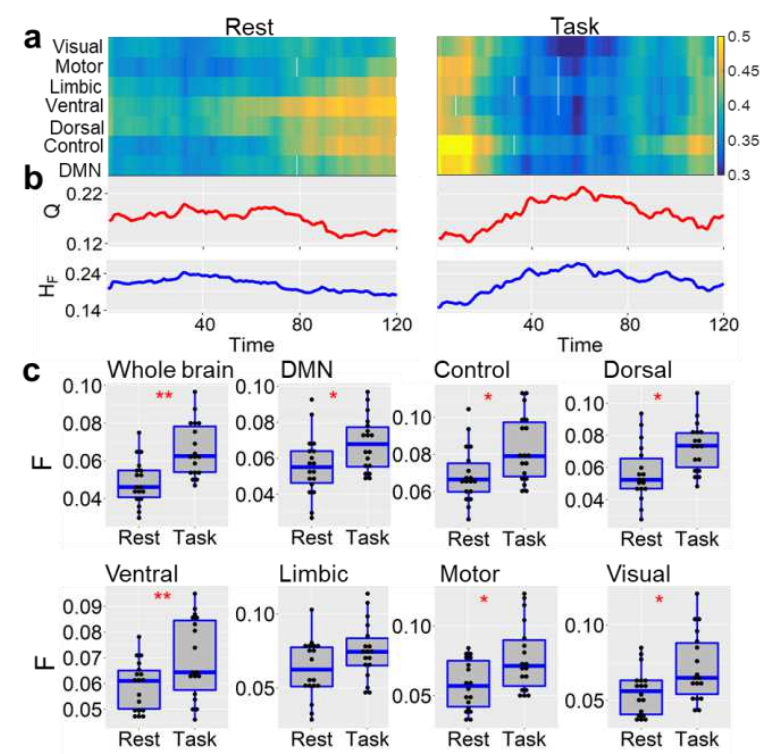

Control Dime Dorsal

Figure 4. Greater flexibility in transitions between segregated and integrated states in the

task state. (a) The temporal activation patterns of different functional subsystems during resting and task states, averaged across participants, wherein node degrees were averaged over the cortical regions belonging to the same functional subsystem to measure the activation level of the subsystem. (b) Dynamic modularity $\left(Q\right.$ )and $H_{F}$, which are significantly anticorrelated with the degree of activation, are more variable during task performance than during rest. (c) Comparisons of functional flexibility between resting and task states for the whole-brain network and seven functional subsystems, wherein “*” is a significant difference (two-sample t-test, $p<0.05$, FDR 
corrected) and “**” represents $p<0.01$.

\section{Functional flexibility predicts cognitive performance}

Since the resting analysis can reveal the 'intrinsic'/context-independent functional organization of the brain and has been linked to many cognitive abilities ${ }^{9,13,20,40}$, we next tested whether the intrinsically flexible transition in functional organization during rest can predict human cognitive performance. The intrinsic functional flexibility $F_{\text {Rest }}$ in the resting state for the whole-brain network was significantly related to $\mathrm{RT} /(\operatorname{Stdev}(\mathrm{RT})$ ) (Figure 5a), and it was also statistically significant for the DMN subsystem (see Figure 5d and Supplementary Figure 6). More importantly, the intrinsic flexibility $F_{\text {Rest }}$ in both the whole-brain network and DMN were negatively correlated to RT/(Stdev(RT)) (Figure 5a and Supplementary Figure 6), providing the insight that the flexible transition between segregated and integrated states during rest constrained the cognitive ability of individuals, and brains with higher intrinsic flexibility supported better human behavioral performance.

Cognitive tasks require the fast reconfiguration of functional organization to match the functional demand, and the dynamic transition between segregated and integrated states during task performance may be an important signature for cognitive performance. The functional flexibility $F_{\text {Task }}$ in the task state for the whole-brain network was significantly related to RT/(Stdev(RT)) (Figure 5b), and it was also statistically significant for dorsal attention, limbic and visual subsystems (Figure 5e). Meanwhile, the correlation between $\mathrm{RT} /(\operatorname{Stdev}(\mathrm{RT}))$ and $F_{\text {Task }}$ was significantly higher in these functional subsystems than that in the whole-brain network, indicating that the localized functional patterns captured more dynamic phenotype of human behavior due to the robust activation of local neural circuits during MSIT performance. Conversely, the functional flexibility $F_{\text {Task }}$ of these subsystems and whole-brain network was positively correlated to RT/(Stdev(RT)) (Supplementary Figure 6), revealing that brains with higher functional flexibility in the task state were associated with worse cognitive performance.

Brains with more efficient switching from resting to task states were shown to be associated with higher cognitive abilities ${ }^{9,13,20,40}$, and thus, it was important to subsequently investigate the correlation between changes in functional flexibility and cognitive performance. The differences 
in flexibility $\Delta F=F_{\text {Task }}-F_{\text {Rest }}$ measures switching efficiency in brain functional organization from resting to task states, and a smaller $\Delta F$ reflects more efficient switching. The differences in flexibility $\Delta F$ was extremely correlated to the $\mathrm{RT} /(\operatorname{Stdev}(\mathrm{RT}))$ in the whole-brain network (Figure 5c), and it was also significant in the DMN, dorsal attention, ventral attention, limbic and visual subsystems (Figure 5f), indicating that the differences in flexibility $\Delta F$ was a robust biomarker for human cognitive behavior. More importantly, the differences in flexibility in the whole-brain network and significant subsystems were positively correlated to the RT/(Stdev(RT)) (Figure $5 \mathrm{c}$ and Supplementary Figure 6), revealing that the weaker changes in brain functional flexibility in the task state corresponded to better performance. This result provides further evidence that brains with more efficient switching from resting to task states were associated with better cognitive performance.
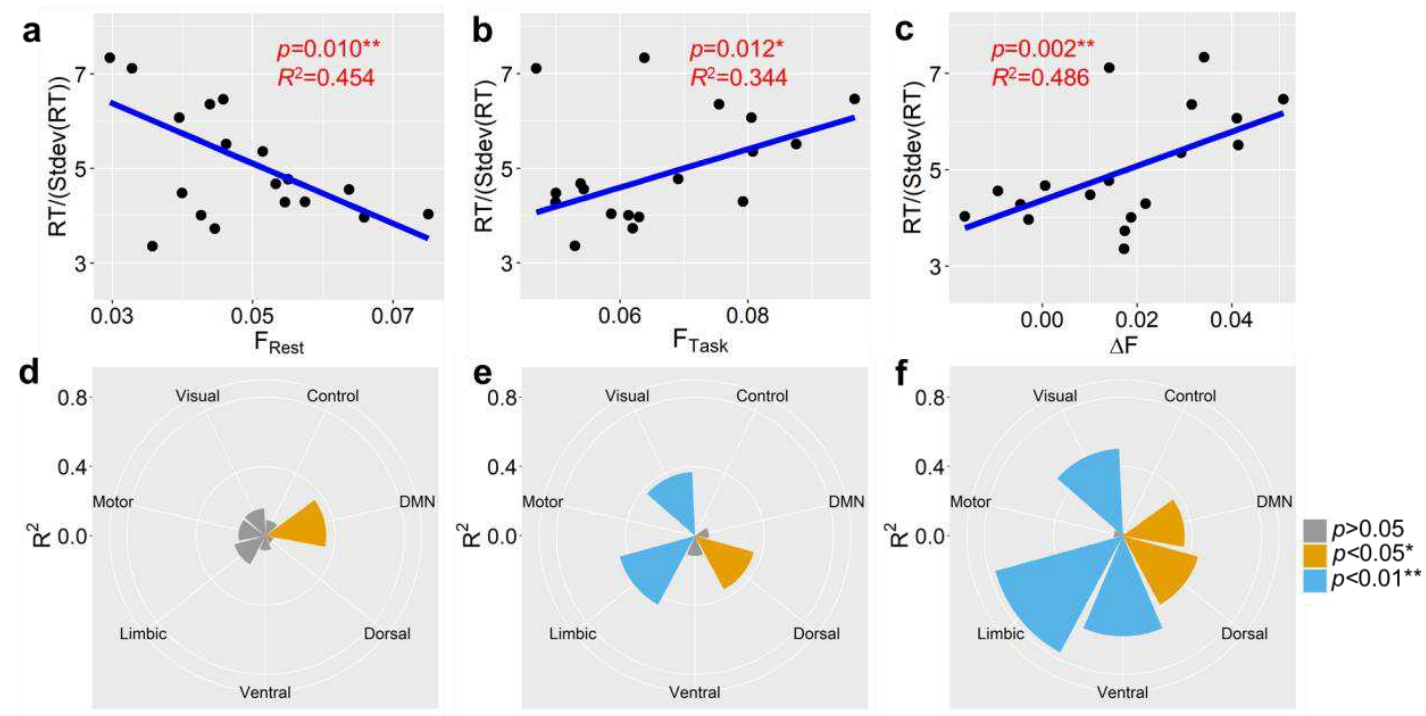

Figure 5. Functional flexibility predicts human cognitive behavior. The relationship between $\mathrm{RT} /(\operatorname{Stdev}(\mathrm{RT}))$ and (a) intrinsic flexibility $F_{\text {Rest }}$, (b) task flexibility $F_{\text {Task }}$ and (c) difference in flexibility $\Delta F=F_{\text {Re } s t}-F_{\text {Task }}$ in the whole FC network. (d) The correlations $R^{2}$ between $\mathrm{RT} /(\operatorname{Stdev}(\mathrm{RT}))$ and $F_{\mathrm{Rest}}$, (e) $F_{\text {Task }}$ and (f) $\Delta F$ for seven functional subsystems. Here, the $p$-values were first computed from the robust linear regression and then FDR correction was performed.

Notably, when functional flexibility was measured by the standard variance in modularity or participation coefficient, $F_{\text {Rest }}, F_{\text {Task }}$ and $\Delta F$ were not statistically correlated to RT/( $\left.\operatorname{Stdev}(\mathrm{RT})\right)$ 
(see Supplementary Figure 7 and Table 1), indicating that our measures based on hierarchical modules in FC networks more effectively captured phenotypes of cognitive performance than classic graph-based network measures at a single level.

Overall, these results confirmed that, based on the hierarchical modules in FC networks, the flexible brain transition between segregated and integrated states is an effective biomarker for human cognitive behavior. Brains that are inefficient in transitioning between segregated and integrated states during rest require more effort to switch to a task state, and subsequently are associated with worse cognitive performance.

\section{Discussion}

In this study, we investigated the relationship between the dynamic reconfiguration of brain functional organization and human cognitive behavior. We proposed a hierarchical measure of network segregation and integration based on hierarchical modules in brain FC networks. Our results showed that brain FC networks dynamically reconfigure their hierarchical modular organization to generate higher segregation at the global scale and most functional subsystems in the task state. In contrast, the brain control subsystem shows stronger integration. Through a dynamic connectivity analysis, we found that brain functional organization more flexibly transitions between segregated and integrated states at global and local scales during task performance than during rest. In addition, we observed strong correlations between flexible transitions in the brain's functional organization and human cognitive performance. Specifically, intrinsic flexibility in the resting state in the whole-brain network and DMN subsystem was positively correlated with cognitive performance, and flexibility in the task state in whole-brain network, dorsal attention, limbic, and visual subsystems was negatively correlated with cognitive performance. Differences in flexibility between task and resting states in the whole-brain network, DMN, dorsal attention, ventral attention, limbic and visual subsystems were negatively related to cognitive performance. Most importantly, these associations with cognitive performance were not observed with graph-based network measures (i.e., modularity and participation coefficient) at a single level. Our results suggest an effective method to study functional segregation and integration by stressing hierarchical modules in brain FC networks and greatly promote the understanding of the relationship between cognitive functions and dynamical reconfiguration of 
brain functional organization across different brain states.

\section{Hierarchical modules in brain functional organization}

Emerging evidence has suggested that the hierarchical modular structure of brain networks may index changes in neural activity patterns underlying critical aspects of cognition and provide clinically relevant information ${ }^{21-23,25,26}$. There is growing interest in the hierarchical modular structure of brain networks as a new approach to understand information processing across different brain states and disease states. However, the current modular partition approach mainly focuses on one level of analysis ${ }^{27}$, which cannot detect the multiple levels of information contained in brain networks. Recently, we found that network eigenmodes can effectively reflect the pronounced modular partitioning in brain structural networks and artificial hierarchical networks across multiple levels ${ }^{21}$, and the NSP method was successively proposed to detect the hierarchical modules in complex brain networks. With the NSP method, we demonstrated that the hierarchical modules in the brain structural network provide a large capacity for functional diversity $^{21}$. From a methodological viewpoint, the NSP method based on orthogonal eigenmodes has a natural physical foundation that regions within a module at a specific level are likely to be cooperatively activated to generate efficient communication. This is in line with the traditional concept of modules that supports more efficient communication within modules ${ }^{27}$, and as such, our indexes are highly correlated with the modularity and participation coefficients. However, compared to the traditional method at a single level ${ }^{22,23,27}$, the NSP method detects the modules in hierarchical levels that correspond to eigenmodes with different orders. Here, we utilized the NSP method to detect the hierarchical modules in brain FC networks across resting and task states and proposed a novel definition (i.e., $H_{F}$ ) to measure hierarchical segregation and integration. Our measures are more sensitive to the detection of individual subtle changes in functional organization at the task state and more effectively detect the phenotypes of human cognitive behavior than classic graph-based network measures (Figure 5 and Supplementary Figures 6-7). Thus, our method across multiple levels is intrinsically different from graph-based network analysis at a single level. Indeed, brain functional segregation and integration reflect the competition between information communicating processes in local and global networks ${ }^{16,42}$. In the framework of hierarchical modular analysis, global communication is extracted at the first 
level. As the level-order increases, more localized information communication within small modules is formed, allowing higher segregation. This detection of local information communication among smaller modules in deeper levels is intrinsically different from graph-based network analysis at a single level and provides our method with intrinsic advantages to capture the brain signatures of human cognitive behavior. Therefore, this work highlights the important role of hierarchical modules in studying segregation and integration and provides a sufficiently effective method to analyze brain functional organization and its relation to cognitive abilities.

\section{Decreased brain network global integration at the task state}

Brain regions within network modules are tightly connected to support efficient communication, but the brain also needs enough strong information flow between them, which eliminates the modules $^{16,42}$. This competition among modules in brain functional organization is dynamically adjusted to support cognitive functions that involve alterations in functional segregation and integration $^{13,15}$ and allows highly flexible information communication across local and global scales. Indeed, different cognitive tasks have heterogeneous demands regarding segregated and integrated brain network processes ${ }^{13,16,43-46}$. For example, a previous study found increased segregation underlying successful motor execution and increased integration underlying successful working memory ${ }^{8}$. According to Chen and Deem's theory ${ }^{47}$, simple tasks require highly modular architectures, which can be completed in a short time and draw mainly on a specific module (or functional subsystem). Here, we found a higher degree of segregation in the whole-brain network in the task state, which was a result of more hierarchical modules. Furthermore, even though most brain subsystems show higher levels of segregation, the control subsystem had higher levels of integration to maintain stable performance in the task state. The MSIT reliably and robustly activates the cingulo-frontal-parietal regions of the brain implicated in processes of executive attention ${ }^{29,30}$, which are mainly contained within the control subsystem. As a simple cognitive task, the MSIT mainly engages the control subsystem to perform the task, and this consequently produces higher segregation in the whole-brain network. This finding provided further evidence supporting Chen and Deem's theory. In fact, the MSIT has been widely adopted to train the cognitive executive function in attention-deficit/hyperactivity disorder (ADHD) patients $^{48}$ and heart failure (HF) patients ${ }^{49}$. Our work revealed a specific response of the control 
subsystem in the brain and may be helpful for the evaluation of cognitive functions.

\section{Higher flexible transitions during rest support better cognitive performance}

The intrinsic properties of brain functional organization at rest constrain the cognitive performance of human behavior ${ }^{10}$. The resting state analysis, independent of specific tasks, provides outstanding advantages in predicting intrinsic cognitive abilities ${ }^{13,40,50}$. For example, pain-free resting states can predict individual pain sensitivity without applying any painful stimulation, which has implications for translational research and the development and assessment of analgesic treatment strategies ${ }^{50}$. In fact, functional patterns during rest mirror part of task-induced activity patterns and predict behavioral performance ${ }^{13,40,50}$, although the signature is not always obvious due to the lack of specific neural responses to tasks. Here, we found a significant correlation between intrinsic functional flexibility $F_{\text {Rest }}$ in the resting state and cognitive performance, and thus, the flexible transition between hierarchical segregation and integration in functional organization may be another intrinsic signature for cognitive performance. Intrinsic flexibility was positively related to cognitive performance in the whole-brain network, indicating that brains with higher rates of reconfiguration of functional organization in the resting state was associated with better task performance. Indeed, a possible explanation is that brain functional organization in the resting state should be flexibly reconfigurable to quickly switch to the task state to support ongoing task performance ${ }^{51}$. Several studies have found that the reconfiguration of functional organization during rest was positively related to attention level, and the ability to reconfigure can be decreased by high levels of fatigue ${ }^{52}$. In addition, drug-induced arousal also resulted in increased reconfiguration in dynamic $\mathrm{FC}^{53}$, indicating that the normal brain in the resting state may need highly flexible reconfiguration in functional organization. Here, we provided further evidence that the resting brain maintains fast reconfiguration to prepare for better cognitive task performance.

We also found that at the local scale, the DMN was the only functional subsystem with a significant association with human cognitive performance. The DMN is specifically interesting since it is highly active during rest but becomes deactivated during task performance ${ }^{54-56}$. In the resting state, DMN subregions have high degrees of connectivity and actively regulate information communication across local and global scales. This activity is related to many cognitive abilities 
and brain disorders ${ }^{57}$. However, the special role of the DMN in brain cognition is not completely clear and needs ongoing investigation. Here, we showed that the intrinsic flexibility of the DMN was positively associated with cognitive performance, indicating that more flexible information communication within the DMN contributed to better cognitive performance. Furthermore, we found that the DMN and whole-brain network had the most similar levels of flexibility in the resting state compared to other brain functional subsystems (see Figure S8), indicating that the DMN may capture the main signature of functional flexibility in the whole-brain network. As a result, the DMN and whole-brain network have similar significant relationships to cognitive performance. Therefore, our results may indicate that the DMN is crucially important for the resting brain to maintain highly flexible transitions between localized processing and global integrated processing that is necessary for better human cognitive performance.

\section{Higher flexibility in the task state constrains cognitive performance}

As a neural response to tasks, the brain needs faster reconfiguration in functional organization to process information in a timely manner to support better cognitive performance. Previous study has found increased reconfiguration of functional organization in task states compared to the resting state ${ }^{39}$, and we also found a similar result in that the brain in the task state needs faster transitions between segregated and integrated states on local and global scales. Thus, even though functional subsystems have different responses to the task in term of segregation and integration, they all need fast reconfiguration to support cognitive demand. However, higher flexibility may not always benefit cognitive performance. In fact, we observed that brains with higher functional flexibility in the task state had worse cognitive performance. A possible explanation for this phenomenon is that to effectively and stably process the information in the task state, the brain needs to maintain the stability in the dynamic functional organization ${ }^{10}$. Several previous studies have supported this hypothesis. It has been found that brains with more stable dynamic connectivity during tasks were associated with better performance ${ }^{39,41}$, and this stability was higher in the speeded condition ${ }^{39}$. Furthermore, higher stability in the functional organization was related to execution difficulty and better attention abilities ${ }^{40}$ and more intelligence ${ }^{13}$. Here, we found that the global flexibility in the task state is slightly negatively related to cognitive performance, but the functional flexibility in the dorsal attention, limbic and visual subsystems 
was more negatively correlated with cognitive performance. Especially, the functional flexibility in the limbic subsystem was most correlated to task performance (Figure 5E). Indeed, even for a simple cognitive task (MSIT), the subjects still needed a complex neural process to identify when attention was distracted and press the button. Our results indicated that better cognitive performance relies on the stability of information communication around local and global networks, and the dynamic reconfiguration in the limbic subnetwork may most effectively reflect the neural process during task performance.

\section{Efficient state transitions allow higher cognitive ability}

It is a long-standing hypothesis that the resting brain is vigilant and able to switch to a task state in a timely manner to support better cognitive performance. Thus, the more efficient transition from resting to task states is thought to be associated with higher cognitive abilities. This hypothesis has been gradually verified in multiple cognitive tasks ${ }^{9,58,59}$. By using FC similarity between resting and task states to measure the cognitive state transition efficiency, Schultz and Cole confirmed that a more efficient transition corresponded to better performance in language, reasoning and working memory tasks ${ }^{9}$, and Harrewijn et al. also found that a more efficient brain was related to greater bias towards threat in children ${ }^{59}$. This phenomenon has further been observed at the broader scale of cognitive ability, i.e., general intelligence ${ }^{9}$. Furthermore, the efficient state transitions are decreased in older as compared to younger adults ${ }^{60,61}$. Interestingly, we also found that the differences in flexibility between task and resting states was positively related to cognitive performance in the whole-brain network and multiple functional subsystems (Figures 5c, f), indicating that the brain with a more efficient transition from resting to task states supports better task performance. However, previous studies were performed at a single level and did not observe that the correlation between FC similarity and cognitive performance was driven by either the resting state or task state ${ }^{9,59}$. Our analysis was performed at hierarchical levels and found that for the whole-brain network, global flexibility in the resting and task states was associated with cognitive performance (Figure 5). These phenomena were different from previous findings and suggested that high intrinsic flexibility provides the brain with the ability to efficiently switch from resting to task states, and the efficient state transition is driven by functional patterns in both resting and task states. Therefore, our results further enhance the understanding of efficient brains 
in that high flexibility for transitioning during rest allows the brain to switch to task states more easily. As a result, an efficient brain obtains higher stability in functional organization allowing stable, successful performance of cognitive tasks.

In conclusion, our results based on a hierarchical modular structure in FC networks provide a systemic understanding of how brain functional organization dynamically switches between segregated and integrated states to support successful cognitive behaviors in terms of resting and task states. The resting brain is optimized to maintain a state with high levels of functional transitions between segregated and integrated states and prepared to most efficiently transition to the task state. This optimized switch in states allows the brain to stably process neural information during tasks to enhance performance.

\section{Limitation of the study}

The small sample size may be a limitation. Indeed, studies with different sample sizes may extract inconsistent observations ${ }^{62-64}$. Our results provide systemic and reasonable relationships between brain flexibility and human cognitive behavior, and part of the results were consistent with previous findings. However, a larger sample size would be expected to enhance these relationships and reveal more neural signatures of human behavior. The second limitation is the single task (MSIT) that reliably and robustly activates the control subsystem. Thus, the relationship between brain flexibility and human behavior should be examined in the context of more complex cognitive tasks, especially considering the heterogeneity in brain responses to cognitive tasks with different complexity ${ }^{8}$. Additional investigations would be valuable to further elucidate the cognitive mechanisms underlying flexible transitions between different states in the brain. Finally, this work focused on normal people, but brain disorders can change functional patterns ${ }^{37,41}$. It would be interesting to further investigate whether these relationships between brain flexibility and cognitive behaviors are maintained in those with brain disorders.

\section{Methods}

\section{Participations}

Eighteen subjects (mean age: 29.3 years, range: $20-49$ years) participated in the experiment. All subjects were healthy without a history of neurological or psychiatric episodes. The participants 
gave written informed consent, and the protocol was approved by the ethics committee of the First Affiliated Hospital of Xi'an Jiaotong University, China.

\section{Multisource interference task (MSIT)}

The MSIT was performed according to the standard protocol developed by Bush and colleagues ${ }^{30}$, and the detailed procedure can be found in Refs. [29,31]. Briefly, the task involved trials with four numbers $(1,2,3,0)$ shown in the center of the screen. The participants were required to identify the target number $(1,2$ or 3$)$ by pressing one of three buttons, which corresponded to 1,2 and 3 , respectively. There were only three control task trials (100,020 and 003) with distracter zeros, wherein the targets were always placed congruently with their positions among the buttons. The interference trials contained two distracters $(1,2$ or 3$)$, and the target numbers were not placed in positions corresponding to the buttons, such as 232. During scanning, the control and interference trials appeared in an alternating pattern, and each trial lasted for $2 s$. The participants needed to complete 100 trials of each type and were instructed to respond as quickly as possible via buttons. The response times (RTs) were automatically.

\section{MRI data acquisition}

All images were acquired using a 3.0T whole-body scanner (GE Signa HDxt, Milwaukee, WI, USA) equipped with an 8-channel head receiver coil. Head movement was restricted using a pillow and foam, and earplugs were used to minimize scanner noise and maximize patient comfort.

A three-dimensional T1-weighted fast spoiled gradient echo (FSPGR) sequence covering the whole brain was performed to coregister functional data and define regions of interest (ROIs), using the following parameters: repetition time $(\mathrm{TR})=10.8 \mathrm{~ms}$, echo time $(\mathrm{TE})=4.8 \mathrm{~ms}$, flip angle $\alpha=15^{\circ}$, field of view $=256 \mathrm{~mm}$, matrix $=256 \times 256$, slice thickness $=1 \mathrm{~mm}$, voxel size $=1 \times 1 \times 1$ $\mathrm{mm}^{3}, 150$ axial plane, and no gap.

Functional images were acquired by means of a T2-weighted single-shot gradient-echo-planar imaging sequence with the following parameters: $\mathrm{TR}=2500 \mathrm{~ms}, \mathrm{TE}=40 \mathrm{~ms} ; \alpha=90^{\circ}$, field of view $=256 \mathrm{~mm}$, acquired matrix $=64 \times 64$, slice thickness $=3 \mathrm{~mm}$, voxel size $=3.75 \times 3.75 \times 3 \mathrm{~mm}^{3}, 47$ slices, no gap. A total of 150 functional volumes $(375 s)$ in the resting state and 160 volumes (400 
$s$ ) in the task state were acquired. To avoid the potential effect of fMRI length, 150 volumes were used in the final connectivity analysis.

\section{fMRI data processing}

The fMRI data were pre-processed using AFNI (http://afni.nimh.nih.gov/afni/) and FSL (http://www.fmrib.ox.ac.uk/fsl/) according to standard pre-processing protocols ${ }^{32}$, and the first four volumes were excluded from the analysis to ensure the initial stabilization of the fMRI signal. To minimize the motion effects, previous study suggests that each framewise displacement (FD) exceed the $0.5 \mathrm{~mm}$ should be censored ${ }^{65}$. We found that the mean FD across 18 subjects was 0.023 $\pm 0.006 \mathrm{~mm}$ in the resting state and $0.019 \pm 0.006 \mathrm{~mm}$ in the task state, significantly smaller than the standard value $(0.5 \mathrm{~mm})$. Echo-planar imaging (EPI) images were motion and slice-time corrected and spatially smoothed using a Gaussian kernel of $6 \mathrm{~mm}$ full width at half maximum (FWHM). The temporal bandpass filtering $0.01 \mathrm{~Hz}<f<0.1 \mathrm{~Hz}$ was performed to reduce the effects of low-frequency drift and high-frequency physiological noise. In addition, several sources of nuisance covariates were eliminated using linear regression: 1) 6 rigid body motion correction parameters and 2) the signal from the white matter and the signal from a ventricle ROI. Note that the global component of the fMRI fluctuations measured during the resting state is tightly coupled with the underlying neural activity ${ }^{66}$, and the use of global signal regression as a pre-processing step in fMRI analyses remains controversial and is not universally recommended ${ }^{66}$. Therefore, the global whole-brain signal was not removed in this analysis, and some fMRI signals may inevitably have originated from sources other than brain activity and interactions.

The brain was parcellated into $N=90$ regions according to the automated anatomical labeling (AAL) atlas ${ }^{67}$, and each region was regarded as a node in brain FC networks. To perform the dynamic connectivity analysis, the sliding time window method was used to construct temporally dynamic FC networks. The fMRI signals of regions were first divided into 120 windows with a sliding step of $2.5 \mathrm{~s}$ ( 1 point) and a window width of $75 \mathrm{~s}(30 \text { points })^{68}$. Then, the FC between regions was obtained by computing the Pearson correlation coefficient between sliced fMRI time series. Since the global whole-brain signal was not regressed, most connectivity in FC matrices was positive ( $80.13 \%$ in the resting state and $79.88 \%$ in the task state). All dynamic FC matrices in each group were averaged to obtain the group-averaged FC matrices (Figure 1a). The negative 
connectivity in FC matrices was set to zero, and the diagonal elements were kept to one while computing the hierarchical measures.

\section{Nested-spectral partition (NSP) method}

Eigenmode-based analysis has been successfully applied to complex networks ${ }^{21,38,69}$. The FC matrix $C$ can be decomposed into $C=U \Lambda U^{T}$, in which $U$ are the eigenvectors and $\Lambda$ are the corresponding eigenvalues. Due to the larger eigenvalues in FC networks corresponding to a stronger degree of collectiveness in dynamic patterns, the functional modes were sorted according to the descending order of $\Lambda$. The NSP method detects the hierarchical modules in FC networks based on the negativity and positivity of eigenvector values ${ }^{21}$, with the following procedures:

1. In the first functional mode, all regions have the same negative or positive eigenvector value, which was regarded as the first level.

2. In the second mode, the eigenvectors has negative and positive values in regions. The regions with positive eigenvector values were assigned as a module, and the remaining regions with negative values were assigned as the second module. This mode was referred to as the second level. Then, the regions were reordered to obtain the new FC matrix with two significant modules (orders within the module are random).

3. Each module in the second level can be further partitioned into two submodules based on the positive or negative sign of the regions in the third eigenvector, producing the third level. Recursively, the hierarchical modular partition was further performed in higher-order modes until to the level wherein each module contains only one region. During the partition process, the module number $M$ in each level was obtained, and regions were optimally reordered.

\section{Measures of hierarchical segregation and integration}

Hierarchical modules in brain FC networks indicate the hierarchical segregated and integrated processes in a nested manner across multiple levels. At a specific level, regions within a module having the same sign of eigenvector component (e.g., negative or positive) are jointly activated to form the integration among them, whereas the opposite activation (e.g., negative and positive signs) of regions generates the segregation between modules. Thus, integrating small modules at a

high-order level (e.g., $i$ th level) form a large module at the lower-order level (i.e., $(i-1)$ th 
level), which further generates the segregation between this large module and other large modules at this lower-order level. This hierarchically segregated and integrated activity reflected by eigenmodes has the contribution $\Lambda$ to FC networks. The first level in the FC network has only one single module involving all regions, reflecting the global integration to allow effective communication across the whole brain and thus requires the largest contribution. The second level with two modules supports the strong communication and specialized process within two modules and weaker cooperation between them and thus requires less contribution compared to the first global mode. Consequently, higher-order modes with more modules and smaller modular sizes relate to deeper levels of finer segregated process, generating more localized information flow and coordination, accompanied by lower contributions $\Lambda$. Specifically, the levels with the highest module number allow completely segregated process and require very small contributions. Thus, the functional modes with larger module numbers generate stronger segregation and smaller-scale local integration, corresponding to weaker contributions to the functional organization.

Consistent with the traditional concept of modularity, modules at a given level support the segregation between them and local integration within them. A larger module number $M$ (detected from the NSP method, normalized into [0 1 1] by dividing $N$ ) reflects higher segregation and smaller local integration at this level. Since this segregated and integrated process has the contribution $\Lambda$ to the functional organization, we multiplied the module number $M$ and contribution $\Lambda$ to reflect the hierarchical segregated and integrated interactions at each level:

$$
H_{i}=\Lambda_{i} M_{i}
$$

Here, $M_{i}$ is the module number in the $i$ th level, and $\Lambda_{i}$ is the corresponding contribution (i.e., eigenvalue). Real FC networks contained small negative eigenvalues that have little effect on results and thus were set to zero. The hierarchical segregation and integration in FC networks was defined across all levels:

$$
H_{F}=\sum_{i=1}^{N} \frac{\Lambda_{i} M_{i}}{N}
$$

Here, $N$ normalizes $H_{F}$ independent of the network dimension. Note that $H_{F}$ contains all segregated and integrated component and thus is a measure of hierarchical segregation and integration in brain FC networks. 
To extract the hierarchical segregation and integration in functional subsystems, the mapping of $H_{F}$ on each region was first proposed as follows:

$$
H_{F}^{j}=\sum_{i=1}^{N} \frac{\Lambda_{i} M_{i} u_{i j}^{2}}{N}
$$

where $u_{i j}$ is the eigenvector value of the $j$ th region in the $i$ th functional mode. Then, the $H_{F}^{j}$ of regions involved in a functional subsystem were averaged to measure the hierarchical segregation and integration of this subsystem.

\section{Graph-based network measures}

To compare the results between graph-based network measures and hierarchical measures of functional segregation and integration in FC networks, the modularity and participation coefficient were also computed. The modules in FC networks were detected with a modularity maximization method at a single level, which maximizes the modularity quality function ${ }^{27}$. The modularity $Q$ in undirected weighted networks is defined as follows:

$$
Q=\frac{1}{l} \sum_{i, j \in N}\left[w_{i j}-\frac{k_{i} k_{j}}{l}\right] \delta_{m_{i}, m_{j}}
$$

Here, $w_{i j}$ is the connectivity between nodes $i$ and $j, k_{i}$ is the degree of node $i$ and $l$ is the sum of all weights. $\delta_{m_{i}, m_{j}}=1$ if nodes $i$ and $j$ are in the same module; otherwise, $\delta_{m_{i}, m_{j}}=0$. The modularity $Q$ quantifies the degree to which a network is decomposed into densely connected modules, and a larger $Q$ reflects denser modules and higher segregation.

The participation coefficient quantifies the degree to which a node is connected to other nodes across diverse modules; its definition for a node is as follows:

$$
P_{i}=1-\sum_{m=1}^{M}\left(\frac{k_{i}(m)}{k_{i}}\right)^{2}
$$

where $M$ is the module number and $k_{i}(m)$ is the connectivity strength of node $i$ within the module $m$. The participation coefficient $P$ of a network is the average of $P_{i}$ in all nodes. Here, the module number $M$ is detected by the modularity maximization method used in Eq. 4. These network measures were calculated with the Brain Connectivity Toolbox (BCT, https://www.nitrc.org/projects/bct) in MATLAB software, with no additional processing. 


\section{Data availability}

The datasets generated during the current study are available at

https://data.mendeley.com/drafts/j4nr4dgppv.

\section{Code availability}

The code used in this study is available upon request.

\section{References}

1 Yeo, B. T. et al. The organization of the human cerebral cortex estimated by intrinsic functional connectivity. J. Neurophysiol. 106, 1125-1165 (2011).

2 Sporns, O. Networks of the Brain. (MIT press, 2011).

3 Shine, J. M. Neuromodulatory influences on integration and segregation in the brain. Trends Cogn. Sci. 23, 572-583 (2019).

4 Wang, R. et al. Decreased coherence and functional connectivity of electroencephalograph in Alzheimer's disease. Chaos 24, 033136 (2014).

5 Sporns, O. Network attributes for segregation and integration in the human brain. Curr. Opin. Neurobiol. 23, 162-171 (2013).

6 Deco, G., Tononi, G., Boly, M. \& Kringelbach, M. L. Rethinking segregation and integration: contributions of whole-brain modelling. Nat. Rev. Neurosci. 16, 430-439 (2015).

7 Adjari, R. A. et al. Topological measure locating the effective crossover between segregation and integration in a modular network. Phys. Rev. Lett. 108, 428-437 (2012).

8 Cohen, J. R. \& D'Esposito, M. The segregation and integration of distinct brain networks and their relationship to cognition. J. Neurosci. 36, 12083-12094 (2016).

9 Schultz, D. H. \& Cole, M. W. Higher intelligence is associated with less task-related brain network reconfiguration. J. Neurosci. 36, 8551-8561 (2016).

10 Cohen, J. R. The behavioral and cognitive relevance of time-varying, dynamic changes in functional connectivity. Neuroimage 180, 515-525 (2018).

11 Liegeois, R. et al. Resting brain dynamics at different timescales capture distinct aspects of human behavior. Nat. Commun. 10, 2317 (2019).

12 Lin, A., Liu, K. K. L., Bartsch, R. P. \& Ivanov, P. C. Dynamic network interactions among distinct brain rhythms as a hallmark of physiologic state and function. Commun. Biol. 3, 197 (2020).

13 Hilger, K., Fukushima, M., Sporns, O. \& Fiebach, C. J. Temporal stability of functional brain modules associated with human intelligence. Hum. Brain Mapp. 41, 362-372 (2020).

14 Girn, M., Mills, C. \& Christoff, K. Linking brain network reconfiguration and intelligence: Are we there yet? Trends Neurosci. Educ. 15, 62-70 (2019).

15 Bassett, D. S. et al. Dynamic reconfiguration of human brain networks during learning. Proc. Natl. Acad. Sci. USA 108, 7641-7646 (2011).

16 Gallen, C. L. \& D'Esposito, M. Brain modularity: A biomarker of intervention-related plasticity. Trends Cogn. Sci. 23, 293-304 (2019).

17 Zamora-Lopez, G., Chen, Y., Deco, G., Kringelbach, M. L. \& Zhou, C. Functional complexity emerging from anatomical constraints in the brain: the significance of network modularity and 
rich-clubs. Sci. Rep. 6, 38424 (2016).

Shine, J. M. et al. The dynamics of functional brain networks: Integrated network states during cognitive task performance. Neuron 92, 544-554 (2016).

Fukushima, M. \& Sporns, O. Structural determinants of dynamic fluctuations between segregation and integration on the human connectome. bioRxiv (2020).

Machida, K. \& Johnson, K. A. Integration and segregation of the brain relate to stability of performance in children and adolescents with varied levels of inattention and impulsivity. Brain Connect. 9, 711-729 (2019).

Wang, R. et al. Hierarchical connectome modes and critical state jointly maximize human brain functional diversity. Phys. Rev. Lett. 123, 038301 (2019).

Meunier, D., Lambiotte, R., Fornito, A., Ersche, K. D. \& Bullmore, E. T. Hierarchical modularity in human brain functional networks. Front. Neuroinform. 3, 37 (2009).

Bullmore, E. T., Lambiotte, R. \& Meunier, D. Modular and hierarchically modular organization of brain networks. Front. Neurosci. 4, 200 (2010).

Zhou, C., Zemanova, L., Zamora, G., Hilgetag, C. C. \& Kurths, J. Hierarchical organization unveiled by functional connectivity in complex brain networks. Phys. Rev. Lett. 97, 238103 (2006).

Friedman, E. J. \& Landsberg, A. S. Hierarchical networks, power laws, and neuronal avalanches. Chaos 23, 013135 (2013).

Wang, S.-J. \& Zhou, C. Hierarchical modular structure enhances the robustness of self-organized criticality in neural networks. New J. Phys. 14, 023005 (2012).

Rubinov, M. \& Sporns, O. Complex network measures of brain connectivity: Uses and interpretations. Neuroimage 52, 1059-1069 (2010).

Jia, Y. \& Gu, H. Sample Entropy Combined with the K-means clustering algorithm reveals six functional networks of the brain. Entropy 21, 1156 (2019).

Bush, G. \& Shin, L. M. The Multi-Source Interference Task: an fMRI task that reliably activates the cingulo-frontal-parietal cognitive/attention network. Nat. Protoc. 1, 308-313 (2006).

Bush, G., Shin, L. M., Holmes, J., Rosen, B. R. \& Vogt, B. A. The Multi-Source Interference Task: validation study with fMRI in individual subjects. Mol. Psychiatr. 8, 60-70 (2003).

Mao, C. P. et al. Decreased activation of cingulo-frontal-parietal cognitive/attention network during an attention-demanding task in patients with chronic low back pain. Neuroradiology 56, 903-912 (2014).

Lin, P., Hasson, U., Jovicich, J. \& Robinson, S. A neuronal basis for task-negative responses in the human brain. Cereb. Cortex 21, 821-830 (2011).

33 Wang, R. et al. Random matrix theory for analyzing the brain functional network in attention deficit hyperactivity disorder. Phys. Rev. E 94, 052411 (2016).

34 Wang, R. et al. Spectral properties of the temporal evolution of brain network structure. Chaos 25, 123112 (2015).

35 Xia, M., Wang, J. \& Yong, H. Brainnet viewer: A network visualization tool for human brain connectomics. PLoS ONE 8, e68910 (2013).

36 Shine, J. M. et al. Dopamine depletion alters macroscopic network dynamics in Parkinson's disease. Brain 142, 1024-1034 (2019). 
integration and segregation using whole-brain computational connectomics: implications for neuropsychiatric disorders. Phil. Trans. R. Soc. A 375, 20160283 (2017).

Atasoy, S., Donnelly, I. \& Pearson, J. Human brain networks function in connectome-specific harmonic waves. Nat. Commun. 7, 10340 (2016).

Elton, A. \& Gao, W. Task-related modulation of functional connectivity variability and its behavioral correlations. Hum. Brain Mapp. 36, 3260-3272 (2015).

Fong, A. H. C. et al. Dynamic functional connectivity during task performance and rest predicts individual differences in attention across studies. Neuroimage 188, 14-25 (2019).

Fiorenzato, E. et al. Dynamic functional connectivity changes associated with dementia in Parkinson's disease. Brain 142, 2860-2872 (2019).

Chen, Y., Wang, S., Hilgetag, C. C. \& Zhou, C. Features of spatial and functional segregation and integration of the primate connectome revealed by trade-off between wiring cost and efficiency. PLoS Comput. Biol. 13, e1005776 (2017).

Fan, J. et al. Segregation of salience network predicts treatment response of depression to repetitive transcranial magnetic stimulation. Neuroimage Clin. 22, 101719 (2019).

Baniqued, P. L., Gallen, C. L., Kranz, M. B., Kramer, A. F. \& D'Esposito, M. Brain network modularity predicts cognitive training-related gains in young adults. Neuropsychologia 131, 205-215 (2019).

45 Fransson, P., Schiffler, B. C. \& Thompson, W. H. Brain network segregation and integration during an epoch-related working memory fMRI experiment. Neuroimage 178, 147-161 (2018).

Adhikari, M. H. et al. Decreased integration and information capacity in stroke measured by whole brain models of resting state activity. Brain 140, 1068-1085 (2017).

Chen, M. \& Deem, M. W. Development of modularity in the neural activity of children's brains. Biophys. J. 106 (2014).

Capri, T., Santoddi, E. \& Fabio, R. A. Multi-Source Interference Task paradigm to enhance automatic and controlled processes in ADHD. Res. Dev. Disabil. 97, 103542 (2020).

Jung, M. et al. Construct validity of the Multi-Source Interference Task to examine attention in heart failure. Nursing Research 67, 465-472 (2018).

50 Spisak, T. et al. Pain-free resting-state functional brain connectivity predicts individual pain sensitivity. Nat. Commun. 11, 187 (2020).

51 Bassett, D. S. \& Bullmore, E. T. Small-world brain networks revisited. Neuroscientist 23, 499-516 (2017).

James et al. Temporal metastates are associated with differential patterns of time-resolved connectivity, network topology, and attention. Proc. Natl. Acad. Sci. 113, 9888-9891 (2016). Rack-Gomer, A. L. \& Liu, T. T. Caffeine increases the temporal variability of resting-state BOLD connectivity in the motor cortex. Neuroimage 59, 2994-3002 (2012).

54 Raichle, M. E. et al. A default mode of brain function. Proc. Natl. Acad. Sci. 98, 676-682 (2001).

Alves, P. N. et al. An improved neuroanatomical model of the default-mode network reconciles previous neuroimaging and neuropathological findings. Commun. Biol. 2, 370 (2019).

56 Cui, Y. et al. Dynamic configuration of coactive micropatterns in the default mode network during wakefulness and sleep. bioRxiv (2020). 

in neurological and neuropsychiatric disorders: a review. Yale J. Biol. Med. 89, 49 (2016).

58 Li, F. et al. Brain network reconfiguration during motor imagery revealed by a large-scale network analysis of scalp EEG. Brain Topogr. 32, 304-314 (2018).

59 Harrewijn, A. et al. Combining fMRI during resting state and an attention bias task in children. Neuroimage 205, 116301 (2020).

60 Cole, M. W., Bassett, D. S., Power, J. D., Braver, T. S. \& Petersen, S. E. Intrinsic and task-evoked network architectures of the human brain. Neuron 83, 238-251 (2014).

61 Hughes, C., Faskowitz, J., Cassidy, B. S., Sporns, O. \& Krendl, A. C. Aging relates to a disproportionately weaker functional architecture of brain networks during rest and task states. Neuroimage 209, 116521 (2020). van den Heuvel, M. P., Stam, C. J., Kahn, R. S. \& Hulshoff Pol, H. E. Efficiency of functional brain networks and intellectual performance. J. Neurosci. 29, 7619-7624 (2009).

Pamplona, G. S. P., Neto, G. R. S. S., Rosset, S. R. E., Rogers, B. P. \& Salmon, C. E. G. Analyzing the association between functional connectivity of the brain and intellectual performance. Front. Hum. Neurosci. 9, 61-61 (2015).

Kruschwitz, J. D., Waller, L., Daedelow, L., Walter, H. \& Veer, I. M. General, crystallized and fluid intelligence are not associated with functional global network efficiency: A replication study with the human connectome project 1200 dataset. Neuroimage 171, 323-331 (2018). Power, J. D., Barnes, K. A., Snyder, A. Z., Schlaggar, B. L. \& Petersen, S. E. Spurious but systematic correlations in functional connectivity MRI networks arise from subject motion. Neuroimage 59, 2142-2154 (2012).

Schölvinck, M. L., Maier, A., Frank, Q. Y., Duyn, J. H. \& Leopold, D. A. Neural basis of global resting-state fMRI activity. Proc. Natl. Acad. Sci. USA 107, 10238-10243 (2010).

Tzourio-Mazoyer, N. et al. Automated anatomical labeling of activations in SPM using a macroscopic anatomical parcellation of the MNI MRI single-subject brain. Neuroimage 15, 273-289 (2002).

68 Lindquist, M. A., Xu, Y., Nebel, M. B. \& Caffo, B. S. Evaluating dynamic bivariate correlations in resting-state fMRI: a comparison study and a new approach. Neuroimage 101, 531-546 (2014).

69 Newman, M. E. Modularity and community structure in networks. Proc. Natl. Acad. Sci. USA 103, 8577-8582 (2006).

\section{Acknowledgements}

This work was supported by the National Natural Science Foundation of China (Nos. 11802229, $11972275,11772242,31671144)$ and the Outstanding Youth Science Fund of Xi'an University of Science and Technology (No. 2019YQ3-11).

\section{Author contributions}

P.L., Y.W. and R.W. conceived the idea. P.L. designed the MSIT experiment and collected the MRI and behavioral data. R.W. processed the MRI data and proposed the framework of hierarchical 
modular analysis. R.W., X.S. and Z.C. organized the results and plotted the figures. R.W., P.L., and Y.W. wrote the paper.

\section{Competing interests}

The authors declare no competing interests.

\section{Additional information}

Correspondence and requests for materials should be addressed to P. L. 

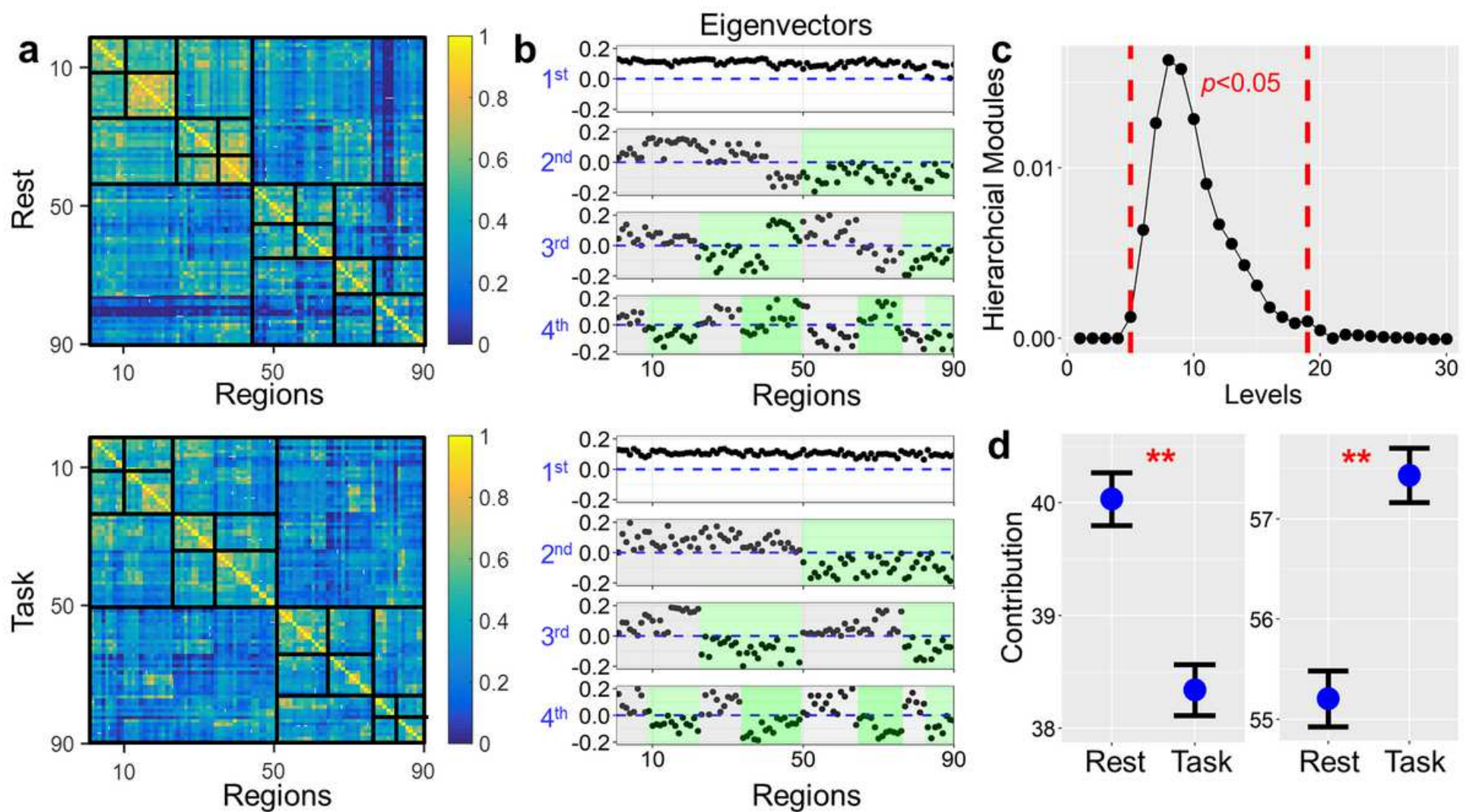

\section{Figure 1}

Hierarchical modular properties of brain functional networks. (a) Hierarchical modular structures of group-averaged FC matrices in resting (upper panel) and task (lower panel) states, detected by the NSP method based on the negativity and positivity of eigenvector values in each level21. The black lines represent the boundaries of hierarchical modules, and the regions were reordered during the NSP process (see Methods). (b) The hierarchical modular partition in the first four functional modes of FC networks in resting and task states, wherein the modules in each level are marked by gray and green colors. (c) The difference in module number between task and resting states in each level across all subjects, where the module number is significantly increased from the 5th to 19th levels (red range; two-sample t-test, $p<0.05$ ). (d) The comparison of the contribution (i.e., eigenvalue) in the first level (left panel) and that summed from the 2 nd to 90 th levels (right panel) in resting and task states. Here, "*ᄎ" represents $p<0.01$ (two-sample t-test). 

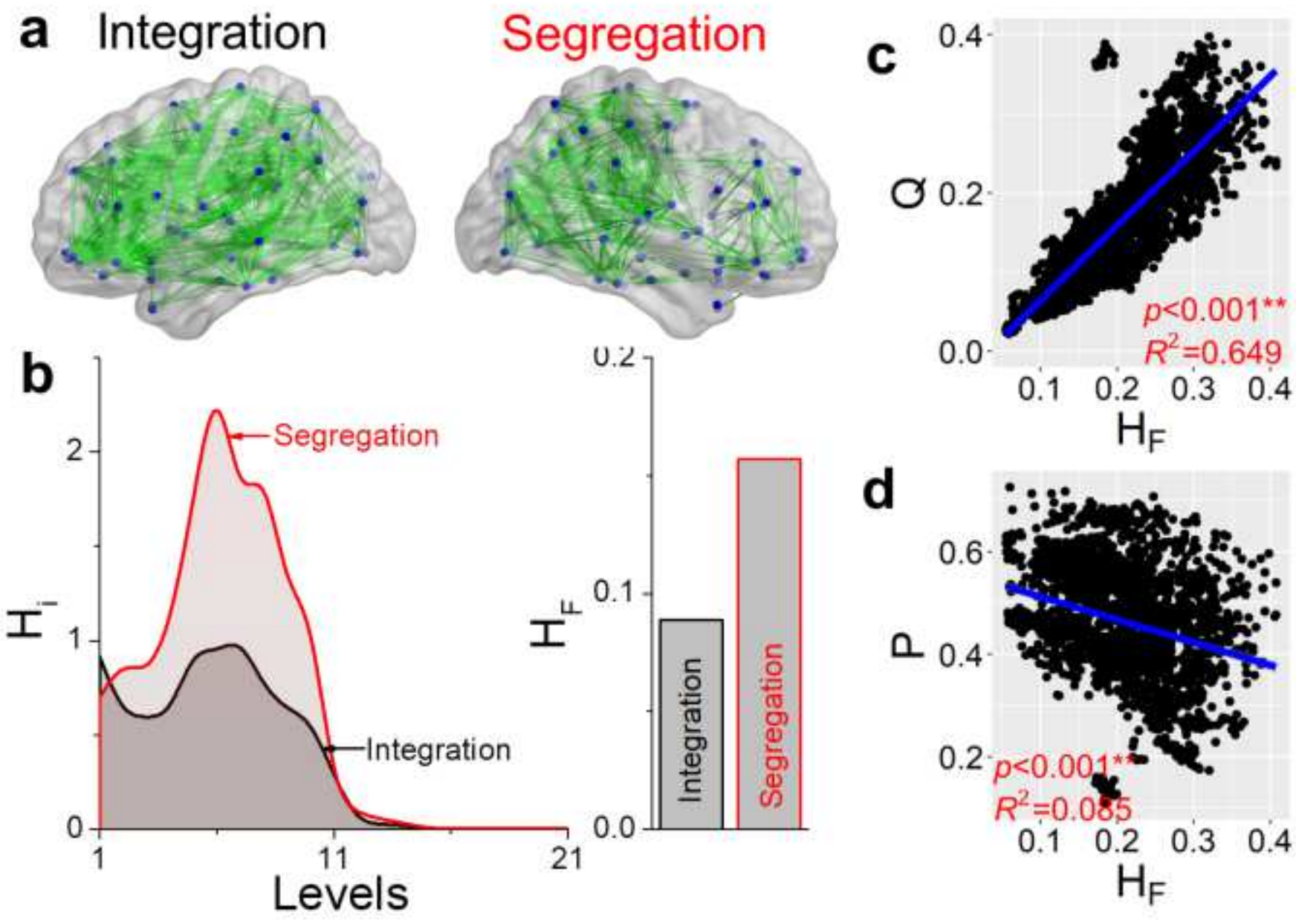

Figure 2

Hierarchical segregation and integration in brain functional organizations. (a) Visualizations of two binarized FC networks in integrated and segregated states using the BrainNet Viewer35. (b) Schematic diagram for the definition of hierarchical segregation and integration. In the FC network, each level generates the segregation and integration, which is measured by the weighted modules (i.e., $\mathrm{Hi}$ ). While the segregated levels with large modules are significantly dominant, the FC network shows higher segregation and sparse connectivity, as shown in a, accompanied by larger HF . (c) The correlations of with modularity $(\mathrm{Q})$ and $(\mathrm{d})$ with participation coefficient $(\mathrm{P})$, which were calculated from FC networks across all temporal windows and subjects in the resting state (see Supplementary Figure 3 for a similar result during the task state). 
a

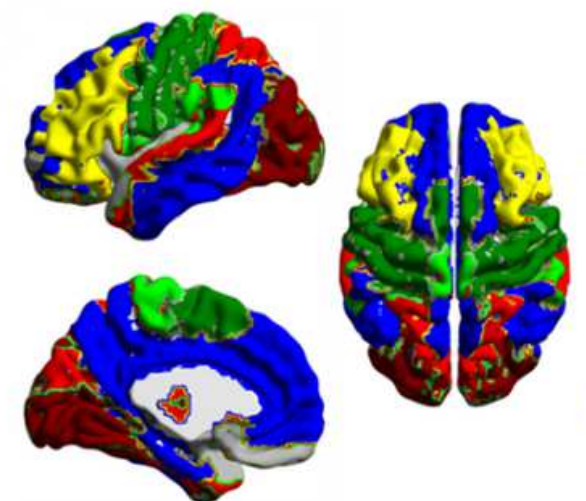

$\square D M N \square$ Dorsal attention $\square$ Limbic $\square$ Visual

$\square$ Control $\square$ Ventral attention $\square$ Motor

b
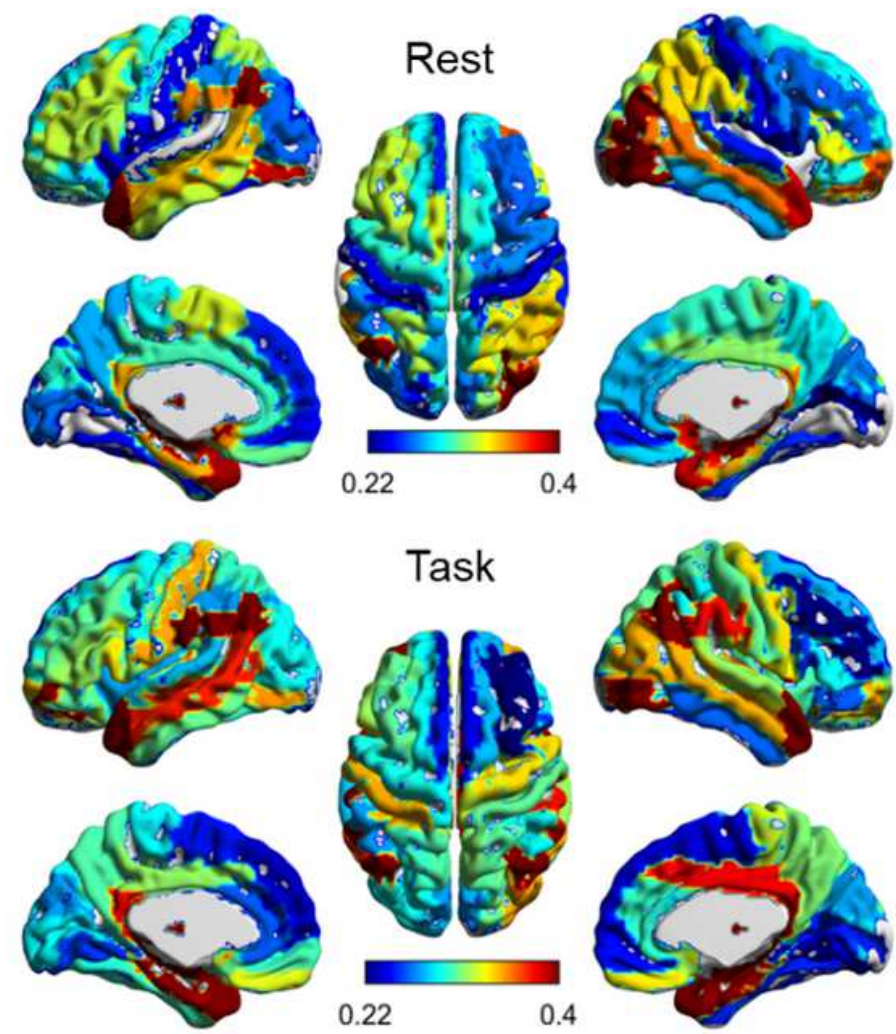
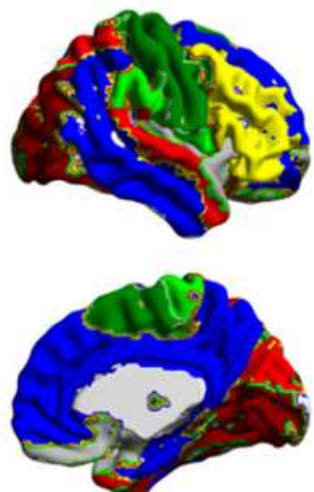
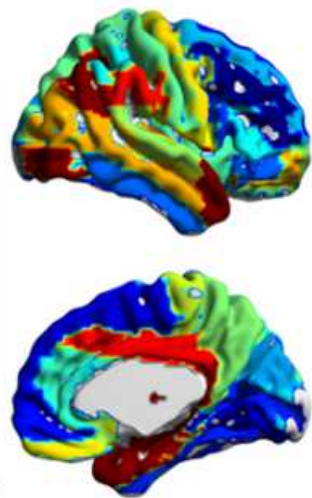

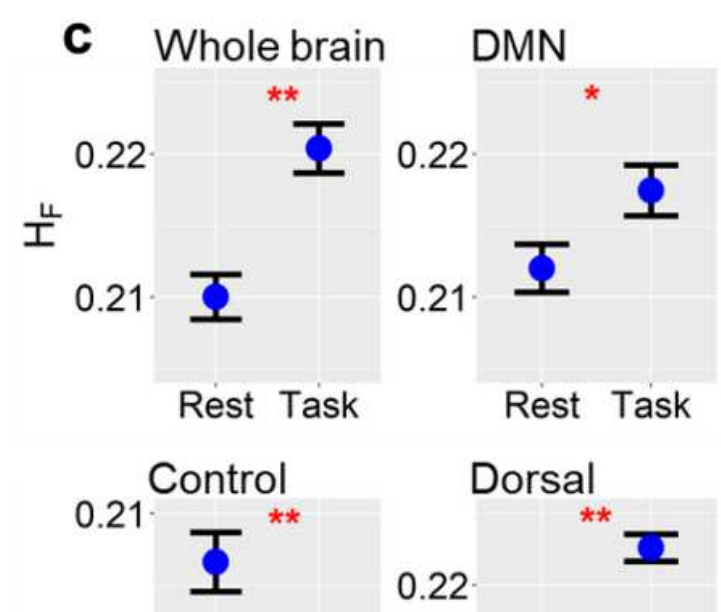

岌 0.20

0.19

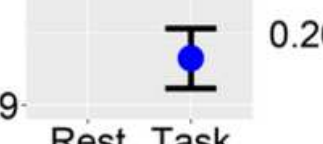

II

Rest Task

Ventral
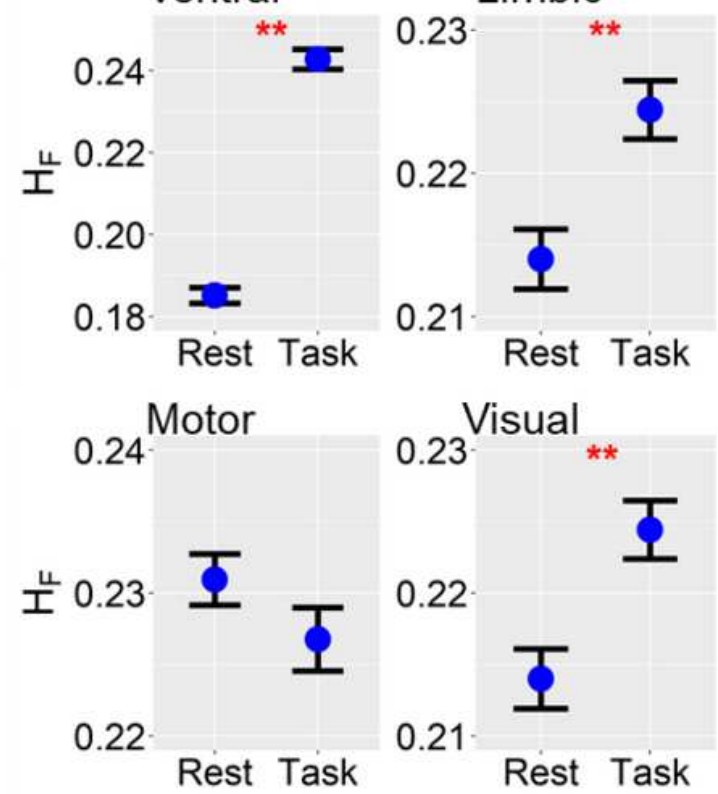

$0.23^{\text {Visual }}$ **

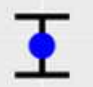

0.22

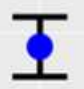

0.21

Rest Task

Figure 3

Alterations in hierarchical segregation and integration in the task state. (a) Brain surface mappings of seven functional subsystems using BrainNet Viewer35. (b) The hierarchical segregation and integration in each region (i.e., $\mathrm{HjF}$, see Eq. 3) in resting (upper panel) and task (lower panel) states, which is averaged across all time windows and subjects. (c) The comparison of HF in the whole-brain network and seven subsystems in resting and task states, wherein " $\star$ " is a significant difference (two-sample t-test,

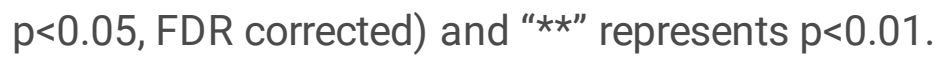



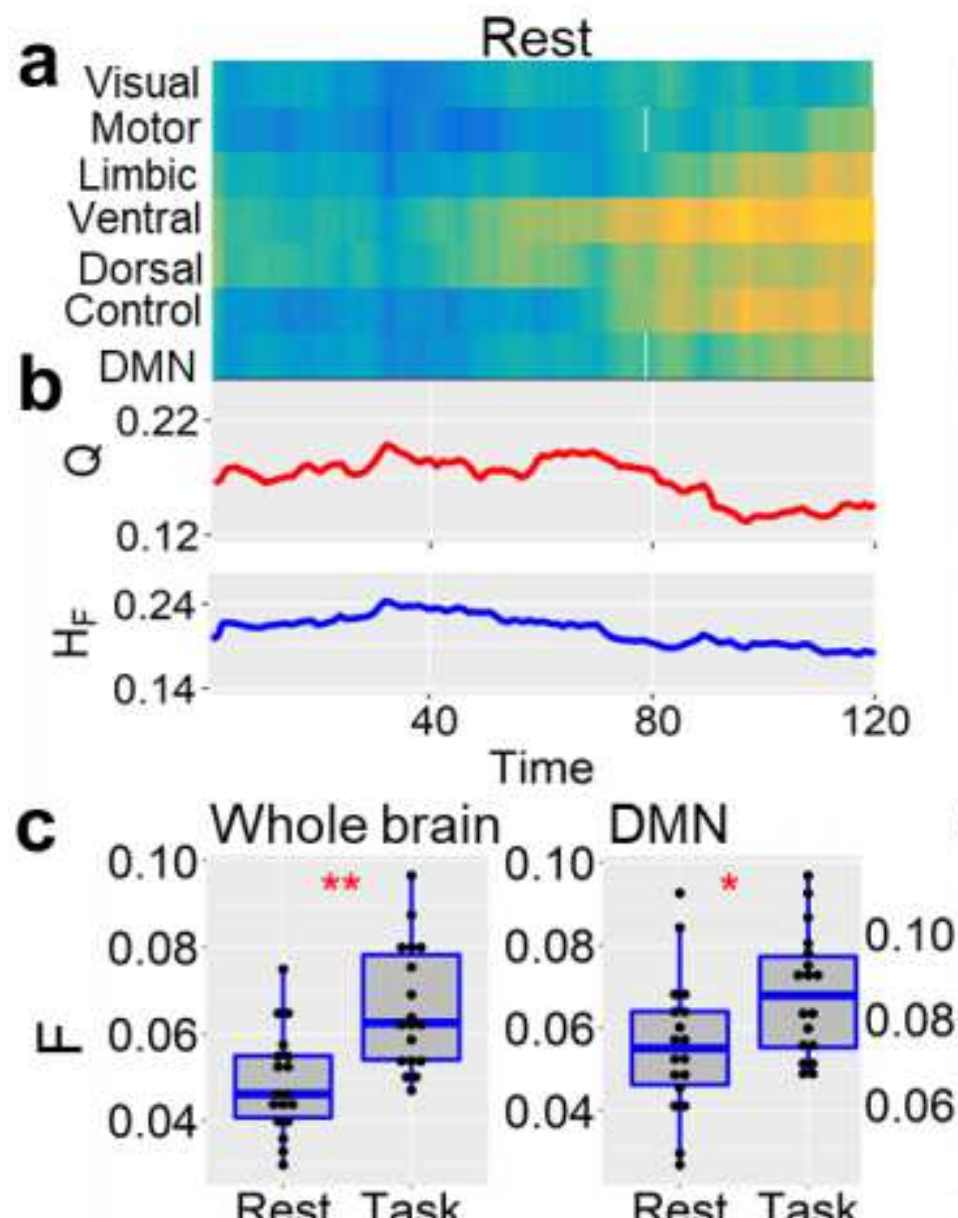

Control
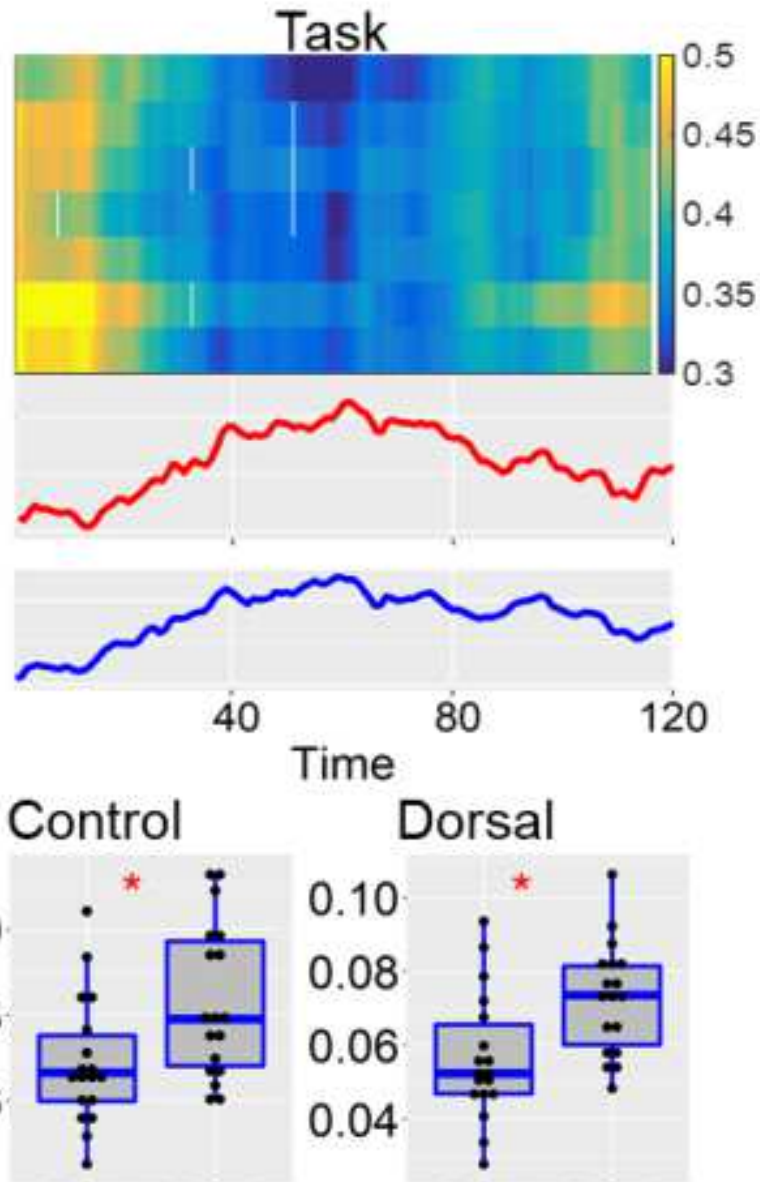

Dorsal

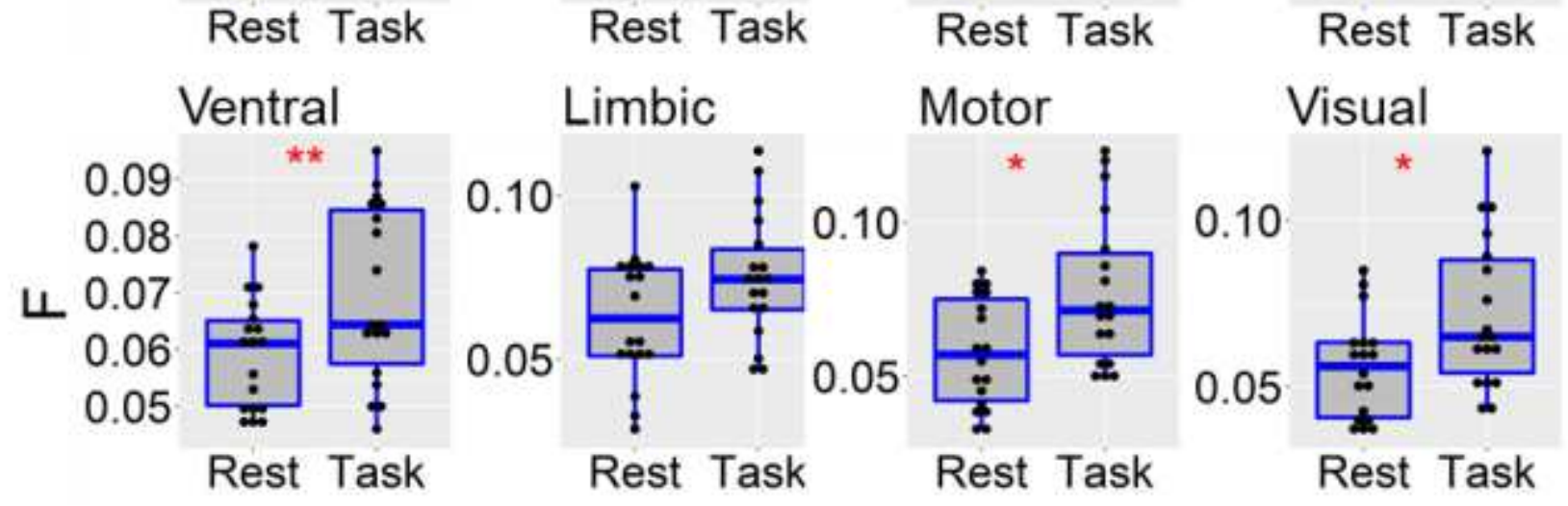

Figure 4

Greater flexibility in transitions between segregated and integrated states in the task state. (a) The temporal activation patterns of different functional subsystems during resting and task states, averaged across participants, wherein node degrees were averaged over the cortical regions belonging to the same functional subsystem to measure the activation level of the subsystem. (b) Dynamic modularity (Q)and $\mathrm{HF}$, which are significantly anticorrelated with the degree of activation, are more variable during task performance than during rest. (c) Comparisons of functional flexibility between resting and task states for the whole-brain network and seven functional subsystems, wherein " $\star$ " is a significant difference (twosample t-test, $p<0.05$, FDR 

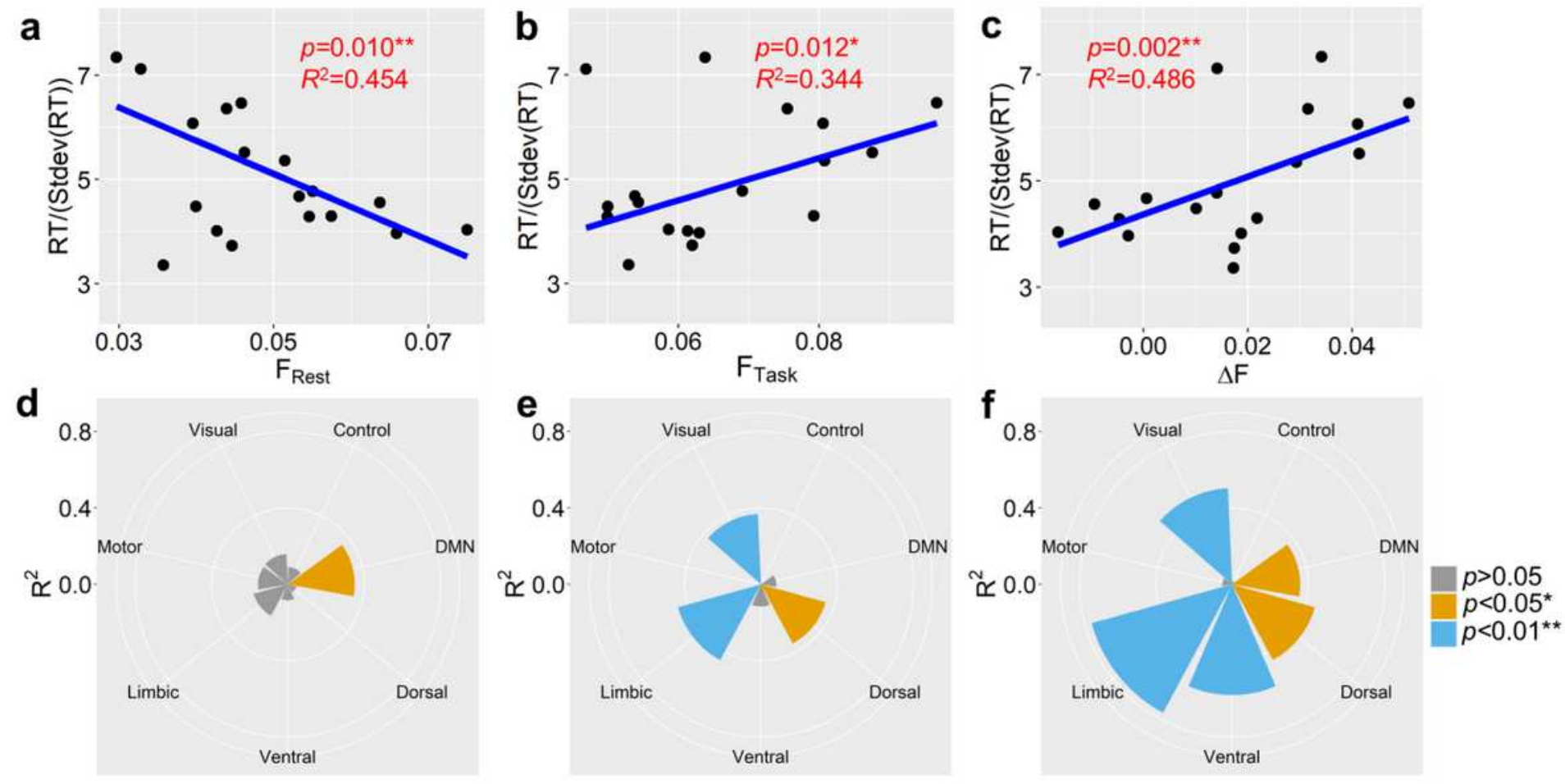

\section{Figure 5}

Functional flexibility predicts human cognitive behavior. The relationship between $\mathrm{RT} /(\operatorname{Stdev}(\mathrm{RT}))$ and (a) intrinsic flexibility FRest, (b) task flexibility FTask and (c) difference in flexibility $\Delta F=F$ Rest - FTask in the whole FC network. (d) The correlations R2 between RT/(Stdev(RT)) and FRest, (e) FTask and (f) $\Delta \mathrm{F}$ for seven functional subsystems. Here, the $p$-values were first computed from the robust linear regression and then FDR correction was performed.

\section{Supplementary Files}

This is a list of supplementary files associated with this preprint. Click to download.

- SIfinal.pdf 